\title{
Clinical ascertainment of health outcomes in Asian survivors of childhood cancer: a systematic review
}

\author{
Long Hin Jonathan Poon ${ }^{1} \cdot$ Chun-Pong $\mathrm{Yu}^{2} \cdot$ Liwen Peng $^{1} \cdot$ Celeste Lom-Ying Ewig ${ }^{1} \cdot$ Hui Zhang ${ }^{3} \cdot$ Chi-Kong Li $^{4,5}$. \\ Yin Ting Cheung ${ }^{1}$
}

Received: 15 December 2018 / Accepted: 5 April 2019 / Published online: 4 May 2019

(C) The Author(s) 2019

\begin{abstract}
Purpose Survivorship in children with cancer comes at a cost of developing chronic treatment-related complications. Yet, it is still an under-researched area in Asia, which shares the largest proportion of the global childhood cancer burden given its vast population. This systematic review summarizes existing literature on clinically ascertained health outcomes in Asian survivors of childhood cancer.

Methods A search was conducted on Ovid Medline and EMBASE for studies that focused on survivors of childhood cancer from countries in East and Southeast Asia; adopted post-treatment clinical ascertainment of organ-specific toxicities or/and secondary malignancy. Studies were excluded if health outcomes were assessed during the acute treatment.

Results Fifty-nine studies, enrolling a total of 13,442 subjects, were conducted on survivors of leukemia (34\%), CNS tumor (14\%), and cohorts of survivors with heterogeneous cancer diagnoses (52\%). The studies used different medical evaluation methods to assess cardiovascular (15\%), metabolic and infertility (32\%), and neurological/neurocognitive (20\%) outcomes in survivors. The collective findings suggest potential differences in the prevalence of certain late effects (e.g., secondary malignancy and obesity) among Asian and non-Asian populations, which may reflect differences in treatment regimens, practice, genetic variations, or/and socioeconomic disparity.

Conclusions We recommend developing collaborative initiatives to build a regional repository of systematically assessed health outcomes and biospecimens to investigate treatment, social-environmental and genetic predictors, and interventions for late effects in this population.

Implications for Cancer Survivors The existing types of chronic health problems identified in this review suggest the need for active screening, better access to survivorship care, and promotion of protective health behavior in Asia.
\end{abstract}

The review was presented at the 8th Nursing Symposium on Cancer Care, Hong Kong (24th to 25th May), and was awarded the Best Poster Award.

Electronic supplementary material The online version of this article (https://doi.org/10.1007/s11764-019-00759-9) contains supplementary material, which is available to authorized users.

Yin Ting Cheung

yinting.cheung@cuhk.edu.hk

1 School of Pharmacy, Faculty of Medicine, The Chinese University of Hong Kong, 8th Floor, Lo Kwee-Seong Integrated Biomedical Sciences Building, Shatin, N.T, Hong Kong

2 Li Ping Medical Library, The Chinese University of Hong Kong, Hong Kong, Hong Kong

3 Department of Pediatric Hematology/Oncology, Guangzhou Women and Children's Medical Center, Guangzhou, China

4 Department of Paediatrics, Faculty of Medicine, The Chinese University of Hong Kong, Hong Kong, Hong Kong

5 Paediatric Haematology \& Oncology, Hong Kong Children's Hospital, Hong Kong, Hong Kong
Keywords Childhood cancer - Late effects - Survivorship · Risk-based · Asian · Organ toxicity

\section{Introduction}

Childhood cancer is an emerging priority in the current global child health agenda. Given its vast population, Asia shares the largest proportion of the global childhood cancer burden. A recent report by the International Agency for Research on Cancer (IARC) indicated that the childhood cancer burden in Asia now accounts for approximately $50 \%$ of all cases worldwide, and this proportion is expected to increase in the coming decades $[1,2]$.

The development of modern treatment strategies has led to tremendous improvements in the 5-year survival and cure rates of childhood cancer during the past 40 years, particularly in high-income Asian countries such as Japan, Singapore, South Korea, and Hong Kong, where survival rates are mostly 
above $80 \%$, comparable to that of the Western population [3-6]. However, survival rates in low-income and middleincome countries of Asia (e.g., mainland China, the Philippines, Indonesia, and Vietnam etc.) can range widely from 20 to $75 \%$, depending on the country, type of cancer, and length of follow-up [7-11]. Although additional research regarding childhood cancer treatment in Asia is unquestionably necessary, efforts should also target the emerging population of survivors.

Cancer survivorship is accompanied by the burden of a myriad of treatment-related complications that can persist for years after treatment completion. A large cohort study of adult survivors of childhood cancer in the USA revealed that at 45 years of age, the subjects had an estimated cumulative prevalence of a serious/disabling or life-threatening chronic condition of $80.5 \%$ [12]. The British Childhood Cancer Survivor Study also reported that among survivors aged at least 60 years, $31 \%$ and $37 \%$ of excess deaths were attributed to subsequent primary neoplasms and chronic cardiovascular conditions, respectively [13]. Substantial evidence in the literature indicates that these chronic morbidities can worsen the emotional health, psychosocial adjustment, and health-related quality of life of survivors of childhood cancer [14-16].

The screening and treatment of late effects in survivors of childhood cancer remain an under-researched area in most regions of Asia, despite gaining recognition as an integral component of the cancer care continuum within North America, Europe, and Oceania. Accordingly, most available data was focused on the health outcomes of survivors in Western countries, with a paucity of clinical data from Asia populations. There is a pressing need to quantify the overall risks of chronic adverse health outcomes in an emerging population of Asian survivors of childhood cancer, as this information will directly affect the allocation of resources and delivery of follow-up care services. These data may also facilitate benchmarking exercises that could eventually direct future research and service planning. This review aims to summarize the existing evidence regarding the clinical ascertainment of treatment-related complications and detection of secondary malignant neoplasms (SMN) in Asian survivors of childhood cancer. It also discusses the rationales behind the urgent need for cancer survivorship research within Asia and directions for future research.

\section{Methods}

A search was conducted on Ovid MEDLINE and EMBASE. Inclusion and exclusion criteria were established to select studies that (1) focused on survivors of childhood cancer, defined as those who were diagnosed with cancer before the age of 19 years and had completed treatment during the time of assessment for complications; (2) used objective clinical assessments to evaluate the endpoints of SMN or/and organspecific toxicities (cardiovascular, pulmonary, musculoskeletal, neurologic, neurocognitive, endocrine/metabolic, fertility, hearing, vision, renal, hepatic, gastrointestinal, hematological, and immunological); and (3) were published in English. In addition, studies must have been conducted on a population of childhood cancer survivors in countries or regions classified as "East Asia" (Mainland China [includes Hong Kong and Macau], Japan, Mongolia, South Korea, and Taiwan), or "Southeast Asia" (Brunei, Myanmar, Cambodia, Indonesia, Laos, Malaysia, the Philippines, Singapore, Thailand, TimorLeste and Vietnam) by major international health and childhood cancer organizations, and consensus from the investigators [17-20]. Due to intrinsic cultural, social, and economic differences among countries across the vast continent of Asia, we did not include countries of the Middle East, South Asia, and Central Asia in the search. In light of the historical development of childhood cancer treatment protocols, this review only includes studies published during or after the year 2000 in order to provide a current perspective on the complications associated with contemporary treatment protocols for childhood cancer. The specific search terms and exclusion criteria are presented in Fig. 1. We also conducted an additional manual search of the references in the manuscripts retrieved.

We did not strictly define the term "survivor" by the duration of time post-diagnosis or post-treatment because of a lack of consensus in the literature and the use of varying criteria in international guidelines. However, studies were excluded if the treatment-related complications were assessed while patients were undergoing active therapy.

Studies with outcomes that were self- or proxy-reported without objective clinical assessments (e.g., quality of life measures and psychosocial measures) were excluded. We included only studies reporting cancer-related toxicities that had been systematically ascertained through formal clinical assessments, rather than including a combination of self- or proxy-reports of the survivors' outcomes as discrepancies among patient self-and proxy-reports, medical records, and findings on clinical examinations are often observed [12, 21]. Meta-analyses, reviews, commentaries, or case reports comprising five or fewer cases were excluded, as were studies that did not describe the basic quantitative research methodology such as data collection methods, clinical assessment methods, and definitions and analytic and/or reporting strategies.

The search results were reviewed on three sequential levels independently by two investigators (LHP and YTC) and resolved through consensus. (1) In the initial "title stage," the article titles were screened to exclude studies that were clearly unrelated to the main interests of this review. (2) In the "abstract stage," the abstracts of articles that passed the "title stage" were reviewed. (3) In the final "full-text stage," the remaining articles were examined to ensure that they fulfilled 
Searches were performed using Medical Subject Heading terms and the free keywords:

("childhood" or "adolescent" or "pediatric") AND ("neoplasms" or "cancer" or "tumor" or "malignancy" or "medulloblastoma" or "leukemia" or lymphoma) AND ("survivor" or "disease free") AND ("brain" or "cerebral" or "respiratory" or "cardiotoxicity" or "cardiac" or "myocardial" or "fibrosis" or "neurological" or "neurotoxicity" or "neurologic" or "neurocognitive" or "cognitive" or "cognition" or "Musculoskeletal" or "bone" or

"skeletal" or "renal" or "kidney" or "endocrine" or "thyroid" or "reproductive" or "gastrointestinal" or "hepatic" or "liver" or "vision" or "visual" or

"hearing" or "auditory" or "dental" or "relapse" or "recurrence" or "deficits" or "necrosis" or "susceptibility" or "morbidity" or "mortality" or "complications" or "outcomes" or "risk" or "comorbidity" or "dysfunction" or "survival rate").

Affiliation of at least 1 author must belong to any of these countries: (China or Japan or Korea or Mongolia or Taiwan or Hong Kong or Brunei or Myanmar or Cambodia or Indonesia or Laos or Malaysia or Philippines or Singapore or Thailand or Timor-Leste or Vietnam) Year of publication restricted to: 1 January 2000 to "current"

Medline $(n=242)$

EMBASE $(\mathrm{n}=406)$

Total (duplicates removed) $(\mathrm{n}=482)$


Fig. 1 Flowchart of literature search

the inclusion/exclusion criteria. After that, the investigators (LHP, LP, YTC) then extracted data independently, peerreviewed each other's summary of the study results, and reconciled any disparities through consensus. The characteristics of the studies were systematically abstracted using a standard methodology with the following parameters: the country in which the study was conducted, publication year, study design, sample size, patient characteristics, and assessment outcomes. Lastly, the quality of each included study was assessed independently using the Quality Assessment Tool for Observational Cohort and Cross-Sectional Studies (LP and YTC) [22]. This tool was chosen as the majority of the reports 
are epidemiology studies which are either observational cohort studies or cross-sectional studies. Inter-rater reliability was calculated using Cohen's kappa statistic to ascertain the agreement between each criterion of the assessment tool as scored by the reviewers [23].

\section{Results}

The results of the literature search are depicted in Fig. 1. The search identified 482 studies from the two databases, of which 188 were excluded at the "title stage." A total of 213 abstracts were reviewed, and 81 full-text manuscripts were subsequently appraised according to the inclusion and exclusion criteria. Finally, the systematic search yielded 59 articles that were included in this review.

\section{General characteristics of studies}

The general characteristics (publication year, country, study design, sample size, cancer diagnoses, treatment modalities, and targeted outcomes) of the 59 studies are summarized in Supplement 1 [24-82]. A total of 13,442 subjects were represented by the included studies. The majority of the studies were conducted in the following developed countries $(n=$ 52, 88\%): Japan [27, 29, 30, 33, 35, 38-40, 42, 43, 45, 49, $54,57,59,61,63,64,67-69,78,80](n=24,41 \%)$, South Korea $[24,26,31,32,34,37,46,53,55,56,58,60,62](n=$ $13,22 \%)$, Hong Kong [41, 50-52, 72-75, 76, 77, 79, 81, 82] $(n=12,20 \%)$, Taiwan $[47,48](n=2,3 \%)$, Singapore [36] $(n=1,1.5 \%)$. Only seven studies $(12 \%)$ were conducted in developing countries [25, 28, 44, 65, 66, 70, 75] (Thailand and Malaysia). Half of the studies focused on either survivors of childhood leukemia $[25,27,28,40,44,46,48,49,58,63,66$, $70,71,73,74,77-79,81,82](n=20,34 \%)$ or CNS tumors $[43,45,47,51,52,60,65,67](n=8,13.5 \%)$, while the remainder included study samples with heterogeneous cancer diagnoses [24, 26, 29-38, 41, 50, 53-57, 59, 61, 62, 64, 68, $69,72,75,76,80](n=29,49 \%)$ The majority of the included studies had sample sizes of $30-150$ subjects $(n=31,52.5 \%)$, with the exception of six studies with larger cohorts $(>500$ subjects) and seven studies with less than 20 subjects. Most studies reported the survivors' mean duration of follow-up, which ranged from 5 to 15 years after the completion of cancer therapy.

The vast majority of studies were epidemiological and explicit about the main objectives of reporting the prevalence and risk factors of the following organ-specific toxicities: cardiac [72-80] $(n=9,15 \%)$; endocrine, fertility, and metabolic [53-71] $(n=19,32 \%)$; neurologic [43-45, 49, 51, 52, 81, 82] and neurocognitive $[46-48,50](n=12,20 \%)$ and SMN [36-42] $(n=7,12 \%)$. Clinical endpoints such as dental [33-35], hepatic [31, 32], renal [30], and immunological
$[28,29]$ outcomes were less commonly reported (combined $n=8,14 \%)$. Four studies (7\%) reported the clinical endpoints of multiple organ systems [24-27].

The assessment of study quality received an inter-rater agreement $k$ of 0.82 . Most studies received a "moderate" $(n=34,57.6 \%)$ or "high" $(n=17,28.8 \%)$ rating (Supplement 2). Due to the criteria stipulated in the literature search framework, all the included studies fulfilled certain recommended qualities of a robust epidemiological study, such as having a clearly stated objective, a specified and defined study population, cancer and its treatment preceding the assessment of late effects, and clearly defined study endpoints that were implemented consistently across study subjects. When considering the individual components of quality, most studies were considered "weak" if they did not consider varying amount of exposure (e.g., dose intensity) or did not adjust statistically for the impact of key potential confounding variables on the association between the treatment and study outcome. Sample size justification is generally not applicable to most studies that are descriptive in nature. A minority of studies $(n=4)$ with radiological assessments as endpoints reported assessments of outcomes blinded to the appropriate members of the research team.

\section{Cardiac outcomes}

Nine studies evaluated cardiac outcomes among Chinese and Japanese survivors of childhood cancer who had been treated with anthracyclines (Table 1) [72-80]. Studies of mixed outcomes (Supplement 1), which included cardiac arrhythmia, cardiomyopathy, congestive heart failure, hypertension, hypotension, and ventricular dysfunction, reported the prevalence of cardiovascular morbidities to be between 3.5 and $16 \%$ [24-27]. Most of these studies had adopted comprehensive and advanced assessments of cardiac function, such as threedimensional and speckle tracking echocardiography, as well as biomarkers of cardiac injury. Cheung et al. and Li et al. highlighted the presence of subclinical anthracyclineinduced cardiotoxicity among asymptomatic childhood cancer survivors with normal ejection fractions [72, 73, 77, 79]. At an average of 15 years after treatment with anthracyclines, the survivors exhibited lower global longitudinal and global radial strain values relative to non-cancer controls, which suggests increased myocardial stiffness and impaired ventricular function in the former group [77]. These observations are consistent with studies conducted in Western populations using similar cardiac assessment techniques [83-85]. Authors speculated the prognostic value of incorporating myocardial strain imaging into long-term follow-up screening guidelines to detect early subclinical cardiotoxicity. These collective evidence support continued periodic cardiac surveillance among adult cancer survivors as an essential component of long-term care. 


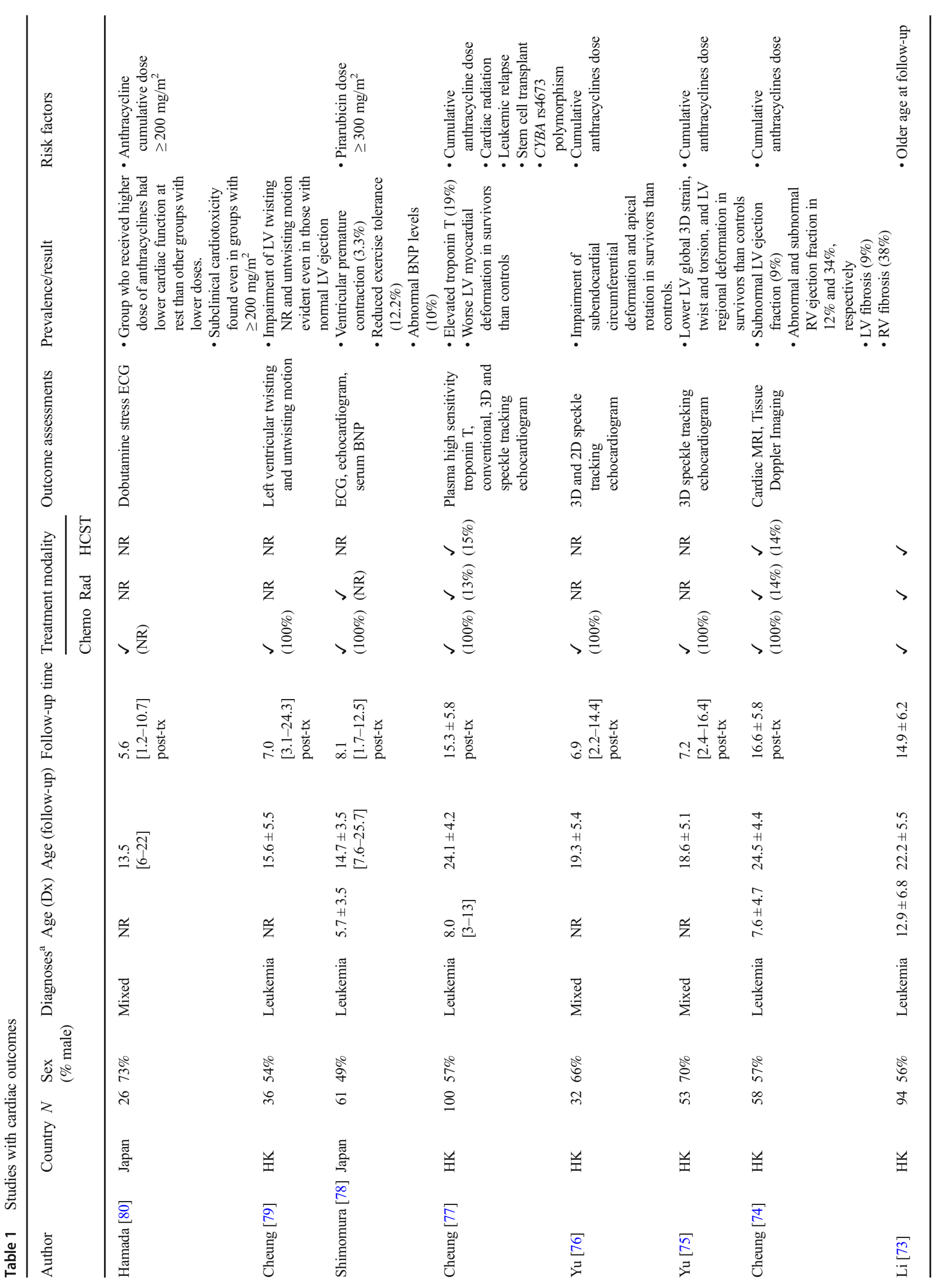


Interestingly, one study observed significantly higher plasma high-sensitivity cardiac troponin $\mathrm{T}$ levels in subjects with the CT/TT genotype at the CYBA gene, which plays a role in physiological free radical metabolism, compared to those with CC genotype [77]. Authors noted that the genotype distributions for this gene differed in the sample of Chinese controls (proportion with variant allele $=21.1 \%$ ), compared to Caucasians $(54.1 \%)[77,86]$. This study provided preliminary evidence that the severity of anthracycline-induced chronic cardiotoxicity may differ across ethnic groups because of genetic variants related to free radical metabolism.

\section{Endocrine, metabolic, and fertility outcomes}

Cancer and its treatments are generally associated with a broad range of complications in the endocrine system that place the survivors of certain childhood cancers at significant long-term risks of obesity, infertility, and metabolic diseases [87].

Most included studies reported a high frequency of disorders affecting hypothalamic-pituitary-adrenal axis, thyroid, and gonadal function, especially among survivors who had been exposed to alkylating agents and/or radiation directed at these organs (Table 2). Deficiencies in growth, thyroid, and follicle-stimulating hormone levels were also detected in survivors [53, 55, 59, 60,63,68,69], and a study of 122 Japanese survivors reported that half experienced gonadal dysfunction [68]. Azospermia and oligospermia were observed in $37.5 \%$ and $12.5 \%$ of male survivors treated with alkylating agents, while a quarter of female survivors required sex hormone replacement [53]. Abnormal levels of sex hormones and gonadal dysfunction (hypogonadism and decreased anti Mullerian hormone, follicle-stimulating hormone, and testosterone) were observed in $34 \%$ to $87 \%$ of participants represented by the studies.

The prevalence of dyslipidemia (i.e., at least one abnormal lipid value, hypertriglyceridemia, or hypercholesterolemia) ranged from 50 to $60 \%$ [57, 62, 67]. Three studies evaluated the prevalence of fatty liver disease in survivors who had received cranial radiation or/and hematopoietic stem cell transplantation [54, 61, 64]. These studies found that even patients who were not overweight or obese developed fatty liver and metabolic abnormalities. Cranial radiation is most commonly associated with the risk of obesity. This association remains consistent in Asian populations, where estimates of the prevalence of obesity among Japanese and South Korean survivors range from 4 to $16 \%[62,64,67,68]$, which is higher than the estimates of $3.0 \%$ to $3.2 \%$ reported in the general population of these two countries [88]. In some included studies, a low lean mass and underweight were endpoints of interest, as they were found to correlate with a lower bone mineral density and higher risk of developing osteoporosis $[58,60,61]$. 


\section{Neurologic and neurocognitive outcomes}

The reported spectrum of nervous system abnormalities includes neurosensory, neurocognitive, and neurologic deficits (Table 3). The majority of the relevant studies included survivors of CNS tumors and pediatric acute lymphoblastic leukemia (ALL) who had received cranial radiation therapy $[43,45$, $47,49-52,81,82]$. Six studies used neuroimaging to identify structural and vascular changes in the brain such as cystic malacia, cavernous angioma, moyamoya, and reduced white matter integrity [43, 45, 49-52]. The prevalence of these neuroimaging abnormalities ranged widely from 10 to $59 \%$ in survivors and was more commonly associated with CNS tumors and higher cranial radiation doses.

Neurocognitive studies identified impairment in the overall and perceptual intelligence in $10 \%$ to $28 \%$ survivors of CNS tumor and leukemia $[47,48,50]$. One study found that half of the survivors demonstrated impaired performance on at least one of the IQ subtests [50]. Few studies have evaluated the neurocognitive outcomes of survivors of other childhood cancers not typically treated with neurotoxic therapies, including neuroblastoma, germ cell tumor, Hodgkin lymphoma, and retinoblastoma.

Whereas most studies focused on CNS toxicities, Tay et al. evaluated the prevalence of vincristine-inducted peripheral neuropathy in survivors of childhood ALL at 2 years posttreatment [44]. The authors found that $15.8 \%$ of the survivors exhibited combined clinical and electrophysiological neuropathy, which was associated with poorer physical functioning [44].

\section{Secondary malignant neoplasms}

The development of SMN is a well-established sequela among long-term survivors of childhood cancer. Among subgroups of survivors, the risk of SMN is related to previous treatment with ionizing radiation, hematopoietic stem cell transplantation, and specific chemotherapies.

All the included studies used in-house medical databases or registries in their countries to identify cases of SMN in survivors of childhood cancer (Table 4) [36-42]. The incidence of SMN remained low even though the risk of secondary cancer remains 6 to 12 times higher than that in the general population. Estimated SMN prevalence rates differ among cohorts of Asian and Western survivors because of differences in the epidemiology of primary cancer diagnoses, treatment strategies, and genetic factors. Interestingly, there are also slight differences in the 20-year cumulative incidences of SMN across Japan [38] (3.2\%), Hong Kong [41] (2.9\%), and Singapore [36] (5.3\%), though these differences may not be clinically relevant and may likely be attributable to the treatment era and exposure to different types and intensities of treatments.

\section{Other outcomes}

Three studies evaluated chemotherapy-induced tooth formation anomalies in survivors with mixed cancer diagnoses (Supplement 3) [33-35]. Approximately 55 to $80 \%$ of survivors developed at least 1 oral or maxillofacial abnormality, especially those who received hematopoietic stem cell transplantation and were treated at a younger age.

Lee et al. and Yoo et al. retrospectively reviewed the clinical and imaging features of hypervascular hepatic nodules in survivors [31, 32]. Both studies concluded that these nodules are benign and unlikely to undergo a malignant change.

Two studies examined the immunological outcomes of survivors $[28,29]$. Azanan et al. reported higher levels of inflammatory cytokines and $\mathrm{T}$ cell responses specific to cytomegalovirus among survivors of childhood leukemia relative to non-cancer controls, while Mahmoud et al. detected chronically elevated levels of Epstein-Barr virus DNA in 20\% of the survivors $[28,29]$. Additionally, Azanan and colleagues discussed the association between the development of agerelated comorbidities and the immune phenotype of aging, as characterized by increased levels of circulating inflammatory cytokines, among survivors [28].

\section{Discussion}

To our knowledge, this is the first systematic review of the available data on the chronic health outcomes among survivors of childhood cancer in Asia. Currently, the majority of survivorship research in the literature originated in North America, Europe, and Oceania, in contrast to the paucity of robust studies from Asia. Our review is unique in that we included only clinical ascertainment of health outcomes that are well-defined and were consistently applied to the populations of each study. We believe that this approach has strengthened the robustness of the review, as reflected by the moderate to high methodological quality of most included studies. Although the studies used different medical evaluation methods and clinical endpoint definitions, the collective findings summarize the existing types of chronic health problems carried by childhood cancer survivors among different ethnic groups across Asia.

Our results suggest that the status of survivorship research within Asia closely reflects the disparities of the available resources. Most survivorship studies in this review were conducted in highly developed countries such as Japan, South Korea, Hong Kong, and Singapore, which share common characteristics such as a high childhood cancer survival rate, established cohorts of childhood cancer survivors, and a high research prioritization of cancer. However, published data and statistics from national registries reveal that up to $70-80 \%$ of children with cancer reside in low- to middle-income 
countries that still struggle with high cancer-related mortality rates [7, 8]. Many countries in Southeastern and South Asia continue to face challenges such as poor access to timely and affordable treatment and a lack of sustainable local pediatric oncology programs [1]. Although continued progress in childhood cancer treatment remains necessary, efforts should also target survivorship care and research among the emerging population of survivors, especially in mainland China and other developing Asian countries. As the susceptibility to and pathogenesis of cancer- and treatment-related chronic health conditions are heavily influenced by genetic factors, environmental exposures, and health behaviors, research efforts must expand to include cases in developing countries.

Importantly, evidence from survivorship studies conducted in Western populations cannot be extrapolated to Asian survivors because of genetic differences in drug responses and susceptibilities to the development of adverse, chronic treatment-associated toxicities. The concept of pharmacoethnicity, which is broadly defined as both genetic and non-genetic ethnic diversity in drug responses and toxicities, is an emerging hypothesis proposed to explain interindividual and inter-ethnic variations in drug responsiveness $[89,90]$. Different rates of chemotherapy-induced toxicities among Caucasian, African American, Asian, and Hispanic cancer populations have been attributed to variations in pharmacogenetics [90-92]. These variations include inherited genetic polymorphisms that render subgroups of cancer survivors more susceptible to certain late effects. For example, one included study identified genetic variations in anthracyclineinduced chronic cardiotoxicity [77], and a high-risk genetic profile for premature menopause was recently identified among childhood cancer survivors who had been exposed to gonadotoxic therapy [93]. Furthermore, inert genetic polymorphisms related to underlying diseases might determine susceptibility to the development of adverse outcomes associated with cancer treatment among survivors. The results of epidemiological studies of general populations suggest that the risk of neurocognitive deficits associated with apolipoprotein E polymorphisms differs among ethnic groups, such that stronger gene-disease associations were observed in Japanese subjects than in Caucasian subjects $[94,95]$. The systematic characterization of the genetic variability in treatment-related complications among Asian survivors is expected to have important clinical implications in this new era of precision medicine.

The non-genetic aspects of cancer pharmacoethnicity include environmental factors and culturally related behaviors, which may have strong influences on drug bioavailability and metabolism [89, 91]. From a biological perspective, the effects of inflammation and oxidative stress on the development of chronic diseases and symptoms in survivors may differ between Western and Asian survivors because of differences in health habits such as physical activity, dietary patterns, and risky behaviors (e.g., alcohol consumption and tobacco use). Although heterogeneity among the study samples in terms of the cancer diagnoses and age at the time of follow-up precludes a meaningful conclusion, our review found that estimates of the prevalence of obesity ( $4 \%$ to $16 \%$ ) among Asian survivors were considerably lower than that observed among Western survivors of childhood cancer. Specifically, the reported prevalence rates of overweight/obesity were $26 \%$ among Swiss survivors of childhood cancer and $26.2 \%$ in the St. Jude Lifetime Cohort Study (USA) [12, 96]. Studies of survivors of childhood ALL have identified associations of biomarkers of vascular injury and inflammation with treatment-induced cardiovascular conditions (hypertension, dyslipidemia, and metabolic syndrome), sleep disturbances, and fatigue [97-99]. Efforts to identify the causative carcinogenic factors and the contributions of environmental and health behavior factors to the development of SMN should maintain the efficacy of contemporary treatments provided to survivors. For example, environmental pollution is known to contribute to cancer epidemiology, the incidence and geographical distribution of SMN in China [100]. These differences might influence the trajectories of late treatment complications during the survivorship phase and should be considered in studies of chronic diseases and SMN in survivors across ethnic groups.

\section{Directions for future research}

This review highlights the need to improve survivorship care and research efforts among Asian survivors of childhood cancer. We propose the following three research priorities:

\section{Opportunities to create a research repository of outcomes data within Asia}

Medical information collected via the prospective systematic screening of late effects in survivors could serve as a framework for future epidemiological and interventional studies unique to the Asian population of childhood cancer survivors. Existing models of survivorship research programs in Western countries could thus be adopted and adapted to spearhead similar initiatives in Asia. One such example is the Childhood Cancer Survivorship Study (CCSS) consortium, which was formed in 1994 by a group of 31 contributing clinical pediatric centers in the USA and Canada [101]. The CCSS is composed of individuals who survived five or more years after diagnosis of childhood cancer and siblings of survivors who serve as the comparison group for the study. The initiation of such a collaborative effort will not merely facilitate the systematic surveillance of survivors and collection of clinical data across different regions of Asia, but will also help to address the research gaps outlined in this review. The collected aggregate information can be used to form a repository 


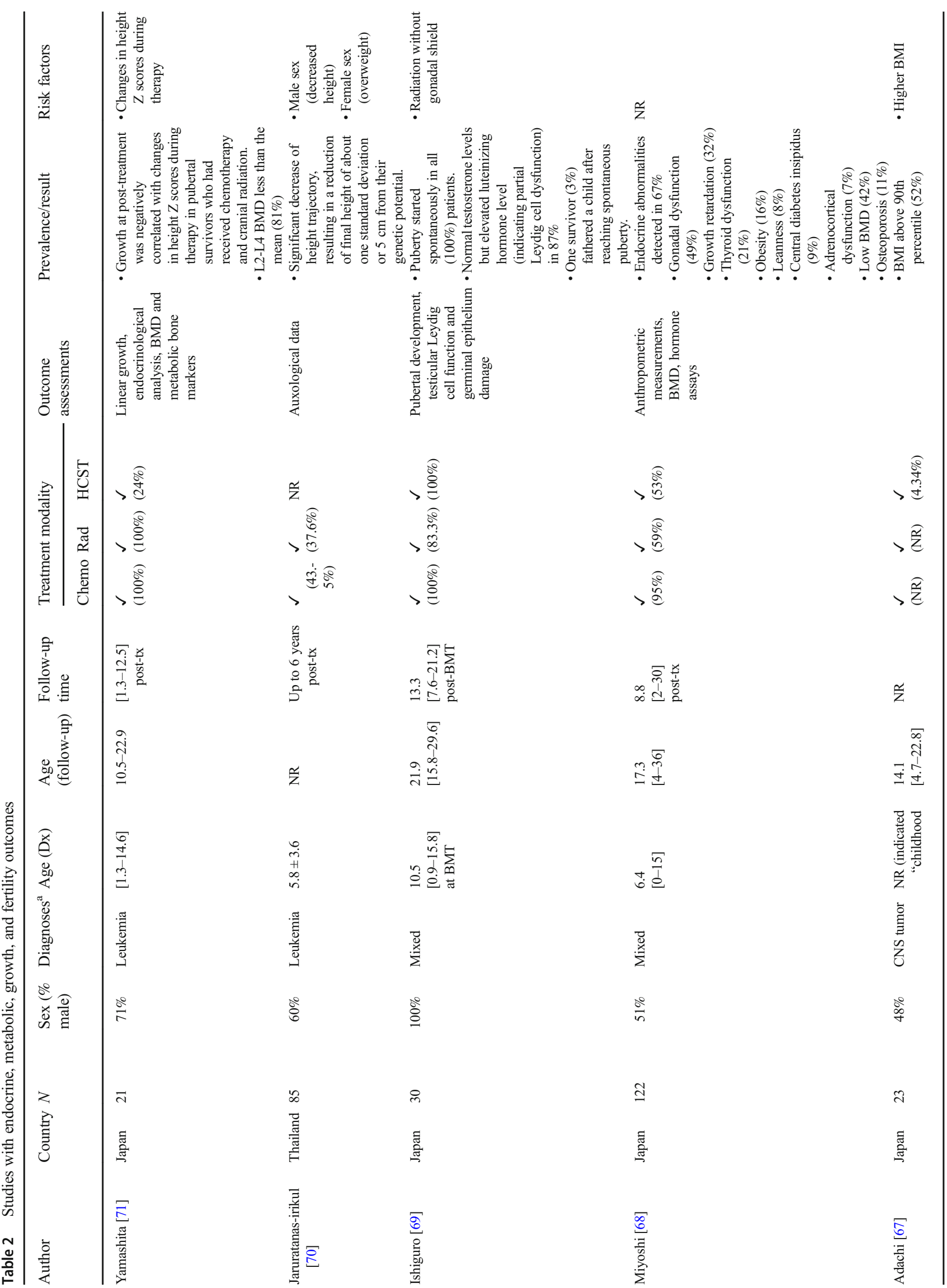




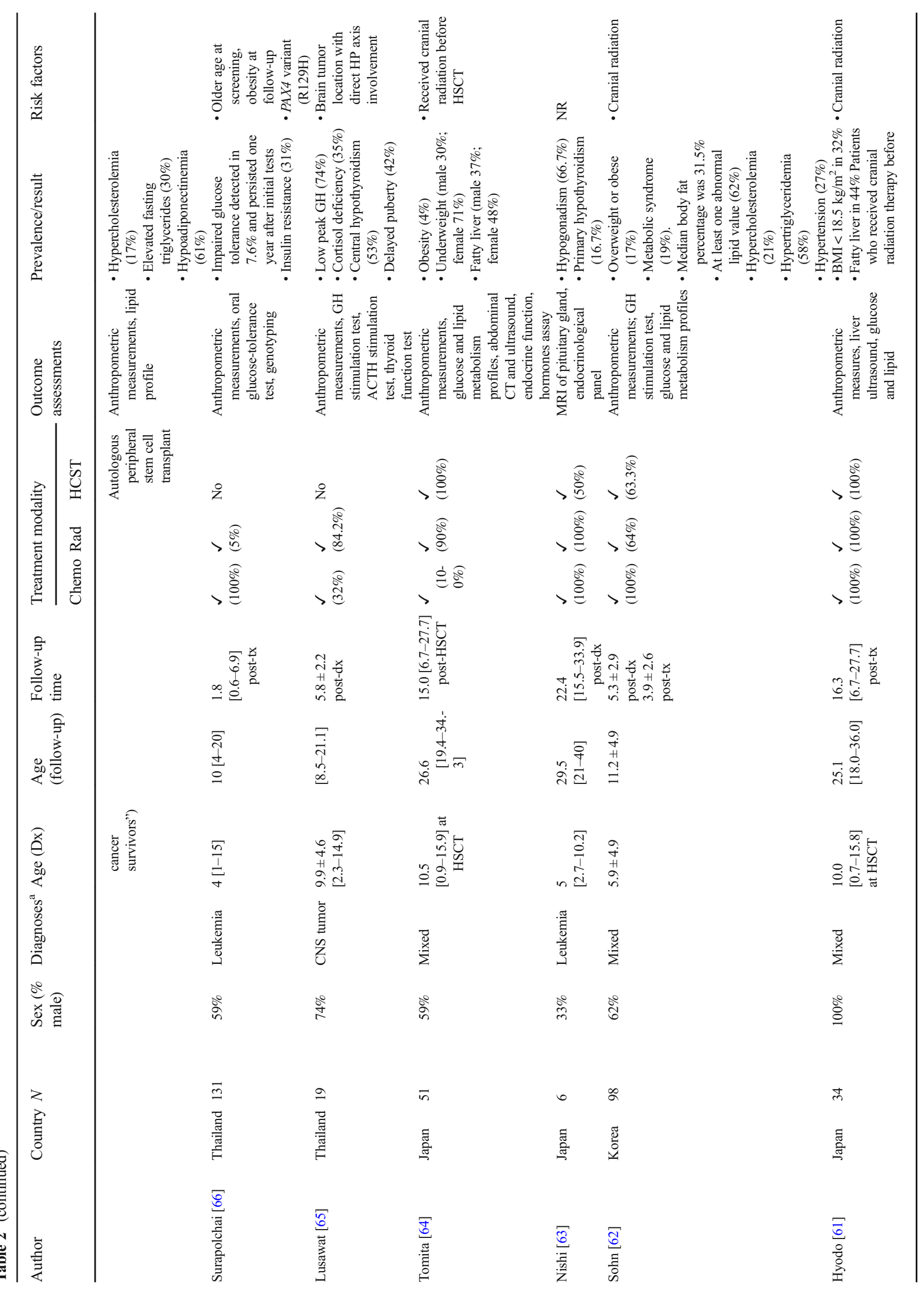




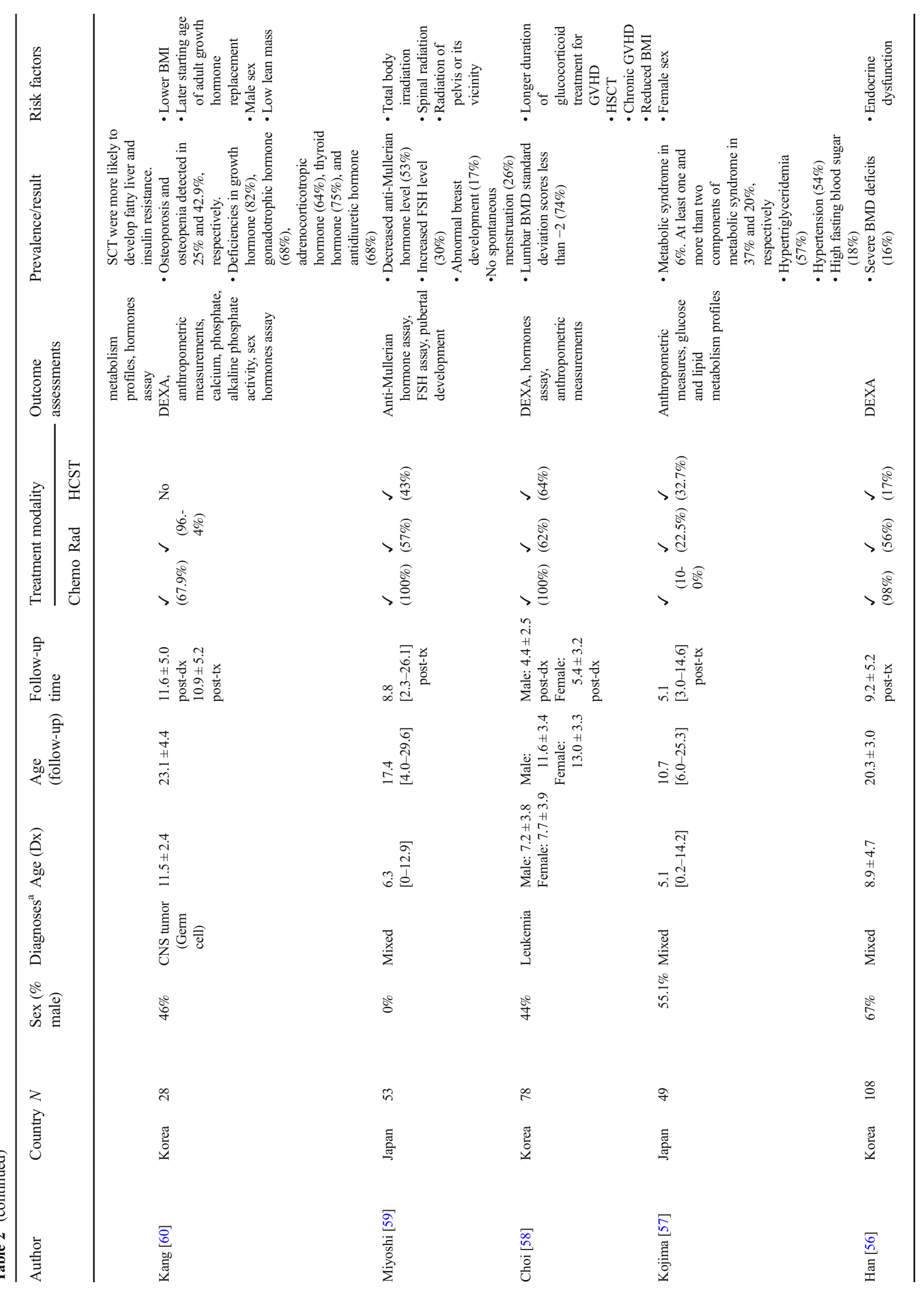




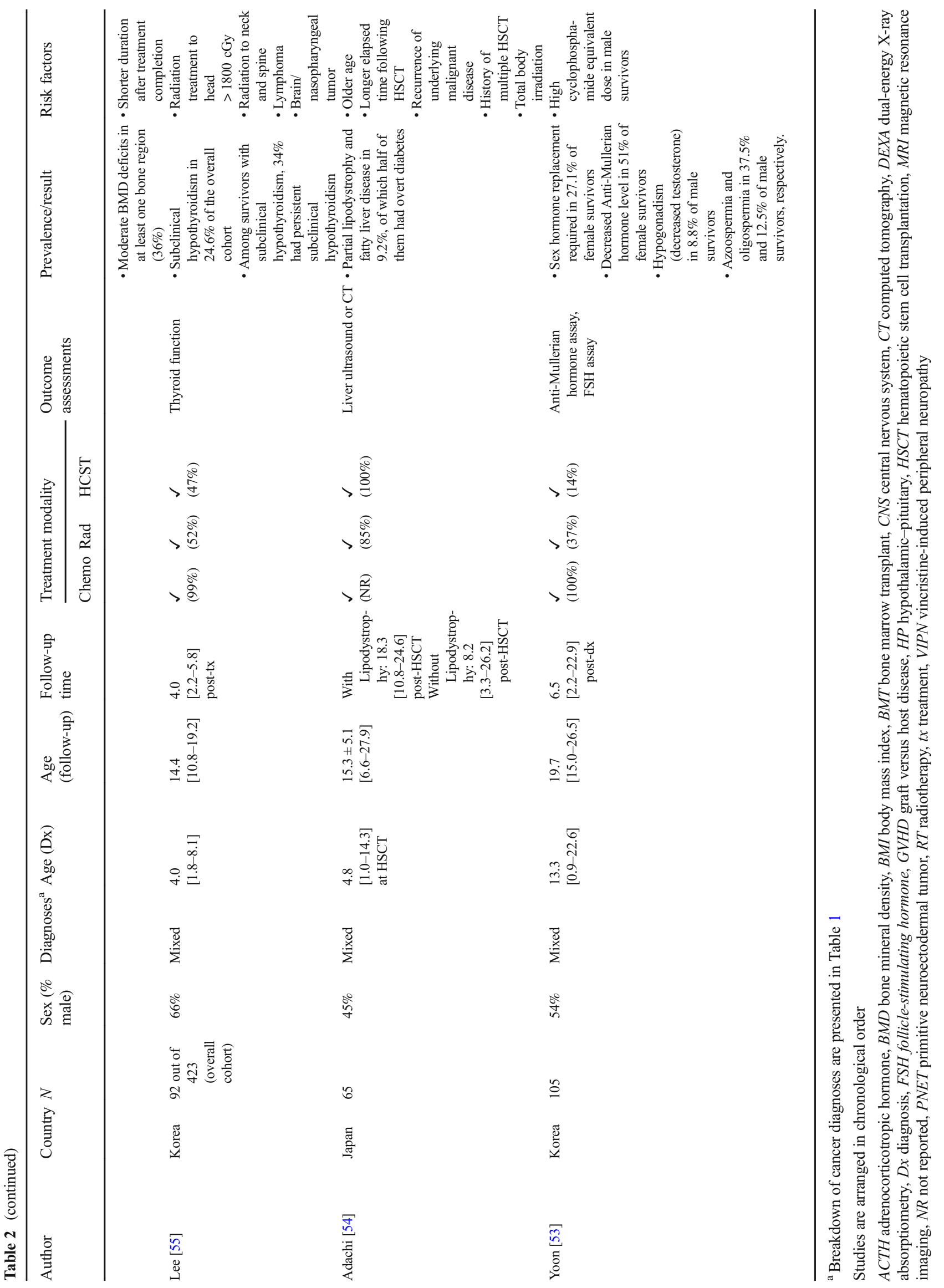


of outcome data that will comprise a valuable research resource readily available to investigators with different professional backgrounds (e.g., medical, nursing, pharmacy, psychology, social work, health services) who are interested in studying the late outcomes of childhood cancer survivors. Notably, multinational studies are often labor-intensive, resource-demanding and likely to involve many investigators and stakeholders, as well as the coordination of study protocols. Existing infrastructures, such as the Asian continental branch of the International Society of Pediatric Oncology (SIOP-Asia) and Childhood Cancer International (CCI), may pave the way for such regional initiatives and collaborative efforts across countries in Asia.

\section{Genetic markers to predict cancer outcomes and late effects}

Studies on both cancer genomes and germline genomes have provided unprecedented insights into the molecular nature of cancer and inter-patient variance [102]. Mounting studies have demonstrated the superiority of machine learning and artificial intelligence in clinical diagnosis and prognosis of childhood cancer [103]. The whole landscape of understanding host and cancer genome plays a crucial role in precision medicine, which will eventually lead to individualized therapies to achieve better response while minimizing long-term toxicities.

Much effort has been initiated on this subject matter over the recent years, particularly in Asia. For example, inherited TPMT and NUDT15 variants are essential genetic markers for mercaptopurine dosage adjustment [104, 105]. Notably, the NUDT15 variant occurs more frequently in East Asians and Hispanics, as compared to Europeans and Africans [104]. Inherited TP53 variants are significantly associated with poorer outcomes and higher secondary cancer incidence in children with ALL [106] while novel ALL subtype (Ph-like ALL) based on gene expression profiling may guide the use of targeted therapies in high-risk patients [107].

Recognizing the power of exploiting genomics data at a population level, China has launched the "100,000 Genomes Project" in 2017, the world's largest genome project, to study the interactions among genetic inheritance, diseases, treatments, and quality of life. However, the overall funding and research involvement for childhood cancer in Asia still substantially lag behind the European and American countries. Applying genetic information to guide the development and selection of childhood cancer therapies in the clinic is promising, yet challenging and requires a multi-national effort. We propose priorities for a research agenda to first pool existing genetic association studies among the Asian cancer population to more accurately quantify the magnitude of risks and functional significance of the variants, and then harness bioinformatics technology to inform reliable prediction models for treatment-related late toxicities. Secondly, research is needed to develop evidence-based guidelines concerning genetic susceptibility testing for late toxicities and implementing them into practice. It is hoped that the current trend toward specialized laboratories, genetic reporting services, and personnel within the Asian region will allow researchers to access gene expression and digitized phenotypic data of patients across national borders.

\section{Novel culturally relevant strategies to prevent or/and treat late effects}

Complementary and alternative medicine (CAM) is broadly defined as medical products and practices that are not part of standard clinical care. Within this broad spectrum of modalities is Traditional Chinese Medicine (TCM). Though the prevalence estimates of CAM use were often evaluated in children with cancer during the active treatment phase [108, 109], it can be reasonable to infer that CAM use may be as prevalent during the survivorship phase, given that TCM is one of the predominating CAM approaches particularly among patients of Chinese ancestry in Asia and worldwide [110].

Despite the growing interest and popularity in CAM use among pediatric patients, the safety and effectiveness of CAM among survivors of childhood cancer are under-researched. Many of these approaches may not be evidence-based due to the difficulty of standardizing the products used and approaches or techniques employed. These impending trends underscore the need for effective models of integrative care that are both evidence-based and culturally sensitive [111, 112]. Further research work evaluating the use of CAM among survivors of childhood cancer may help clarify and answer the many questions and concerns regarding the use of complementary and conventional therapy and better delineate its role in health care needs of childhood cancer survivors.

\section{Implications and recommendations for clinical practice}

\section{Toward the systematic screening of health outcomes}

International working groups, such as the Children's Oncology Group (COG), American Society of Clinical Oncology (ASCO), and International Society of Pediatric Oncology (SIOP), now support the need for evidence-based systematic screening of late effects in survivors with childhood cancer [113, 114]. For example, the "Children's Oncology Group Long-Term Follow-Up Guidelines for Survivors of Childhood, Adolescent, and Young Adult Cancers" includes risk-based, exposure-related clinical practice guidelines that provide recommendations for the screening and management of late effects in survivors [115]. "Risk- 
based" or "exposure-based" care refers to a personalized, systematic plan of regular screening, surveillance, and prevention strategies based on the patient's treatment and cancer history [114]. Such evidence-based guidelines may facilitate a recommendation for the standardized risk-based screening of asymptomatic survivors of childhood, adolescence, or young adulthood cancers who present for routine exposure-related medical follow-up visits.

In every country, existing constraints within the current clinical setting and healthcare system limit the implementation of all aspects of risk-based guidelines. For example, our review found that the neurodevelopmental trajectory is less explored in children with cancer in Asia. This is an important research gap in the literature, especially as educational attainment and academic achievement are highly regarded in most Asian societies. This may be limited by the lack of certified neuropsychologists and developmental psychologists in Asia [116-118]. Therefore, international guidelines must be adapted and modified to meet the needs of the local healthcare structure. Health policy and health system research is needed to weigh other key considerations, which include reimbursement policies for screening tests implemented by the government and private insurance companies, the availability of resources, and labor constraints in the healthcare field.

\section{Improving access to survivorship care}

Primary pediatric clinics often lose survivors of childhood cancer to follow-up because these patients experience increased independence and mobility as they advance into adulthood. Particularly in Asia, the needs of this population are often understudied and under-addressed. Few Asian countries have long-standing programs to provide regular follow-up evaluations and manage the health needs of childhood cancer survivors, and socioeconomic disparities within this region also pose challenges to the abilities of survivors to access such services, even if they are available.

As survivors transit from pediatric to adult care, they face complex issues that require family-centered, inter-related care coordination, and gradual step-down care within the community $[119,120]$. Currently, there is a clear lack of studies evaluating the specific barriers to long-term survivorship care in the pediatric oncology field. However, studies of the barriers to cancer survivorship faced by Chinese breast cancer patients revealed that survivors were wary of entrusting their complex health conditions to primary care physicians, whom they perceived to be poorly equipped to manage cancer-related health problems [121]. One study also reported that Japanese survivors of childhood cancer preferred to receive long-term care from the same physician as adults [122]. Supplement 4 summarizes the potential gaps and barriers to implementing a comprehensive survivorship program based on experiences from European and North American populations [113, 120,
$123,124]$, as well as experience and existing interventions in the Asian population [26, 125-133]. Future work should aim to identify effective and sustainable transition models and create recommendations for and formal evaluations of successful transitional care. These efforts should involve the development of a structured program to facilitate the safe and efficient distribution of clinical services to a multidisciplinary team of primary care providers, allied health professionals, and nongovernmental organizations (NGOs). These programs should also include elements such as financial services, patient navigators, and social workers to help patients prepare for the economic challenges related to cancer and its treatment.

Over the next decade, international and regional collaborations may play an important role in improving access to survivorship care in developing countries. The concept of "twinning," in which a cancer center from a developed country collaborates with another center in a developing country, has been used successfully to improve the childhood cancer survival rates in some countries [134]. Hopefully, such initiatives can be extended to survivorship care within the near future. The two-way transfer of survivorship-related expertise, advice, knowledge, and skills may enhance the construction of infrastructure for survivorship work in developing countries.

\section{Interventions to improve health literacy levels}

Education and empowerment are crucial for survivors of childhood cancer as they assume age-appropriate ownership of their health and become active partners during the survivorship phase [124]. This process includes the promotion of protective health behaviors. Poor health-based knowledge of the importance of detecting treatment-related late complications during the early years of survivorship may affect their health habits during adulthood (e.g., sun protective behaviors, smoking, screening for SMN). A recent study of survivors of childhood cancer in Hong Kong described how their perceptions of fatigue and reduced physical strength after the cancer diagnosis had prevented them from being physically active [135]. Other studies have highlighted that Korean cancer survivors face challenges regarding smoking cessation, as smoking is perceived as a strategy for pain and stress relief $[136,137]$. The promotion of health behavior education among survivors is a timely topic in Asia, where many health and research programs have been implemented to ameliorate the general adverse health effects of the urban environment on children and adolescents, such as sleep disturbances, sedentary lifestyles, and academic stress.

In the USA and Europe, many survivorship programs now incorporate individualized counseling on the personal health risks faced by survivors and caregivers. This includes a review of the survivors' medical and treatment histories to facilitate collaborations among survivors, caregivers, and oncology practitioners in the development of personalized care plans 


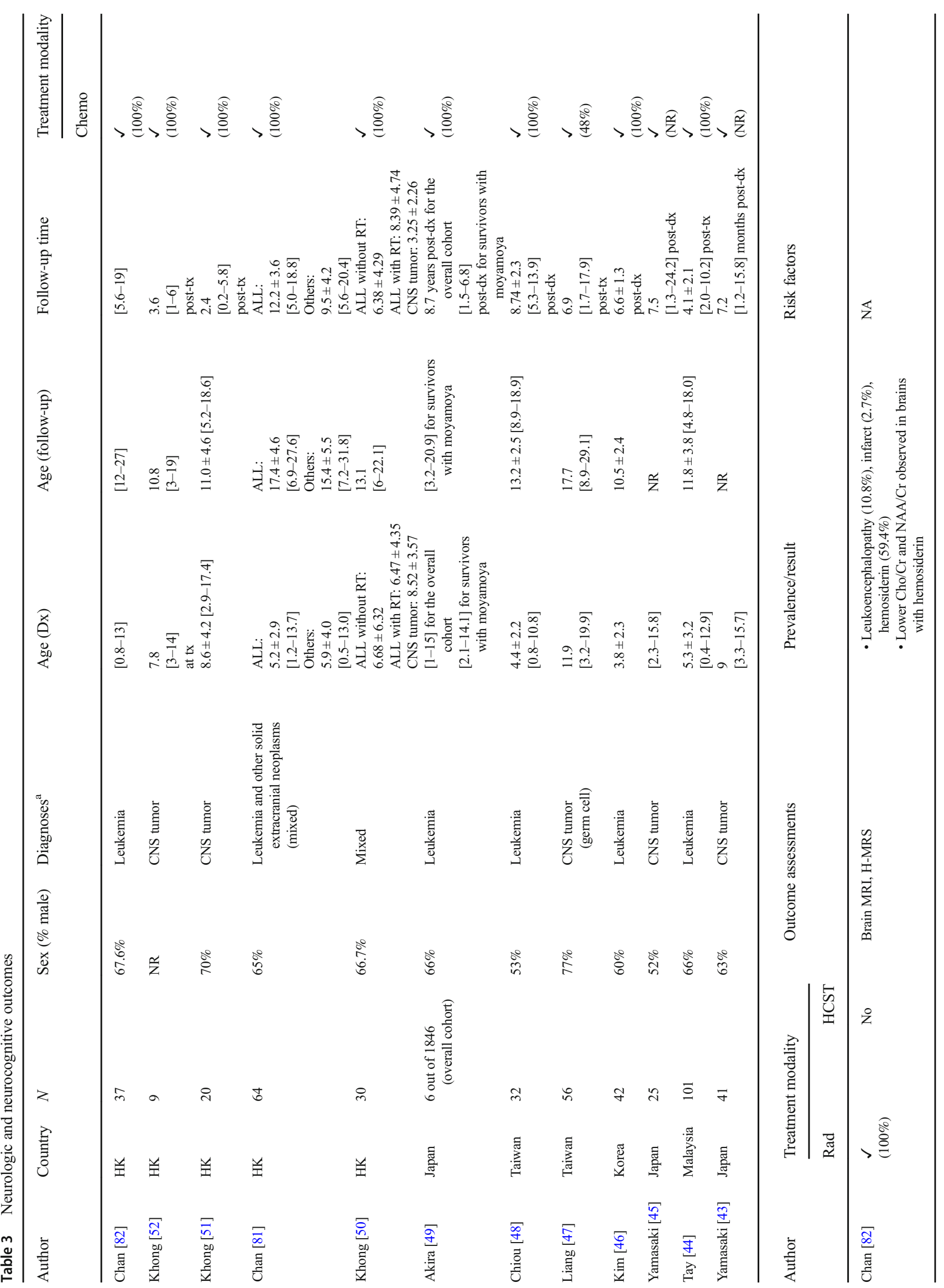




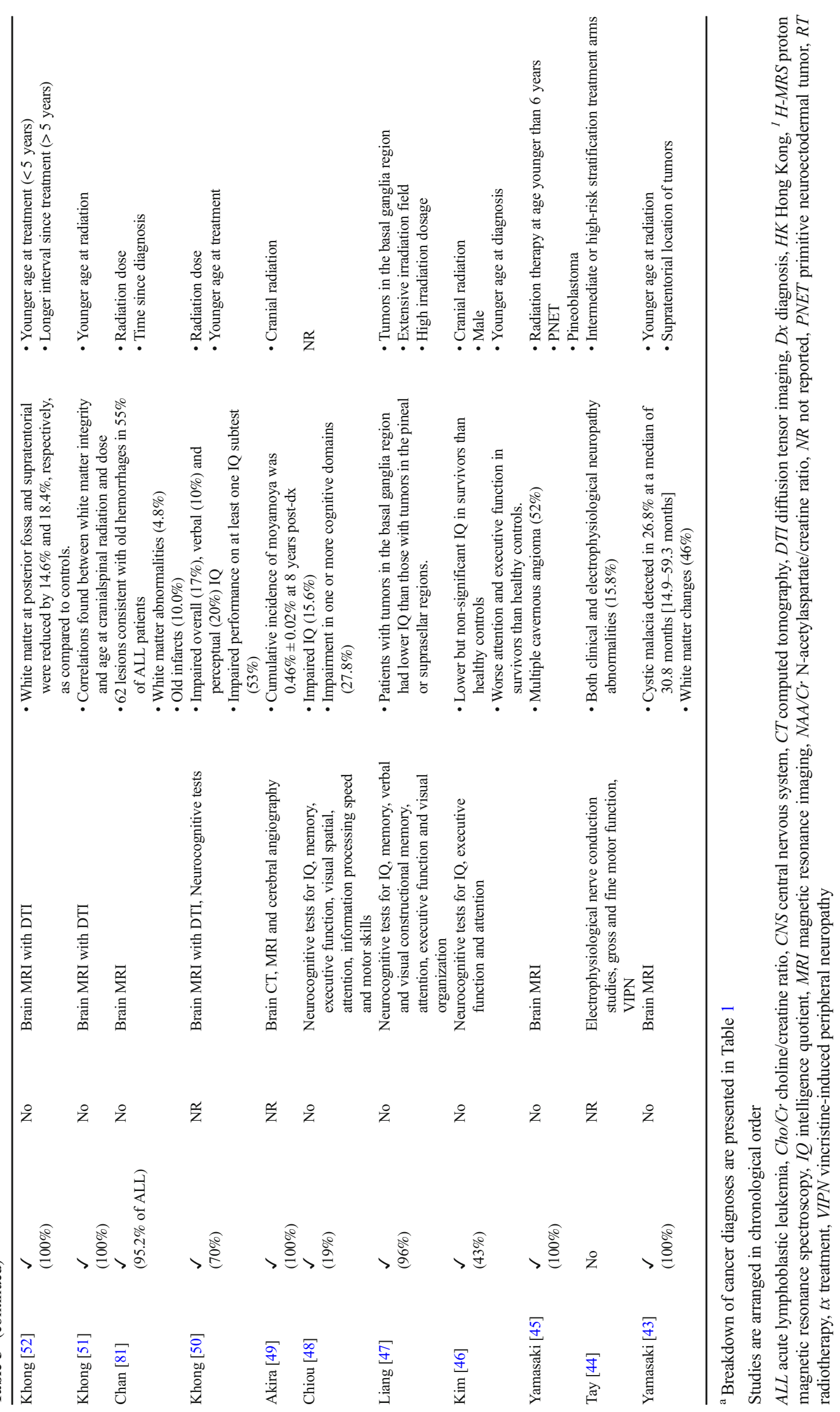




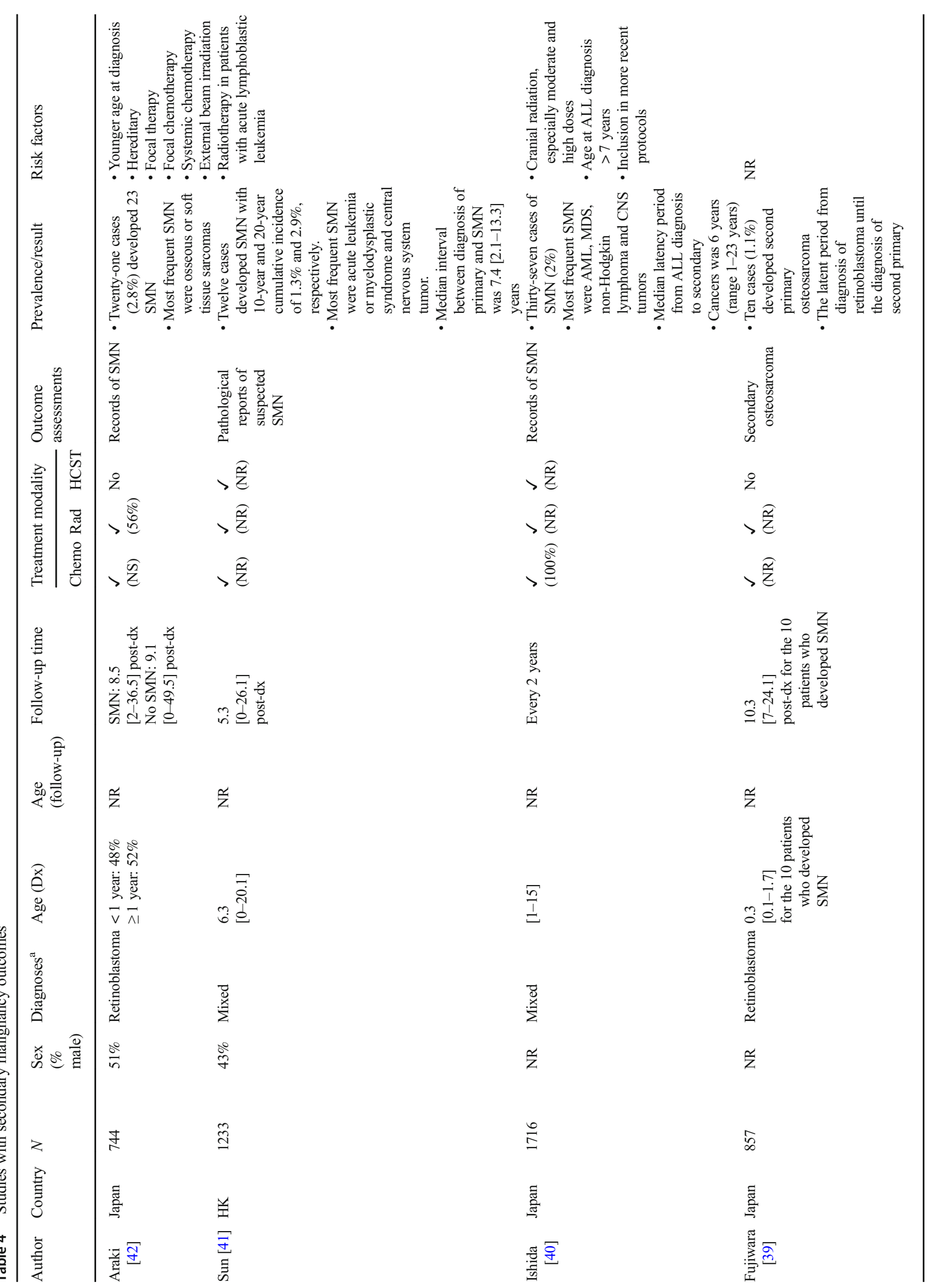




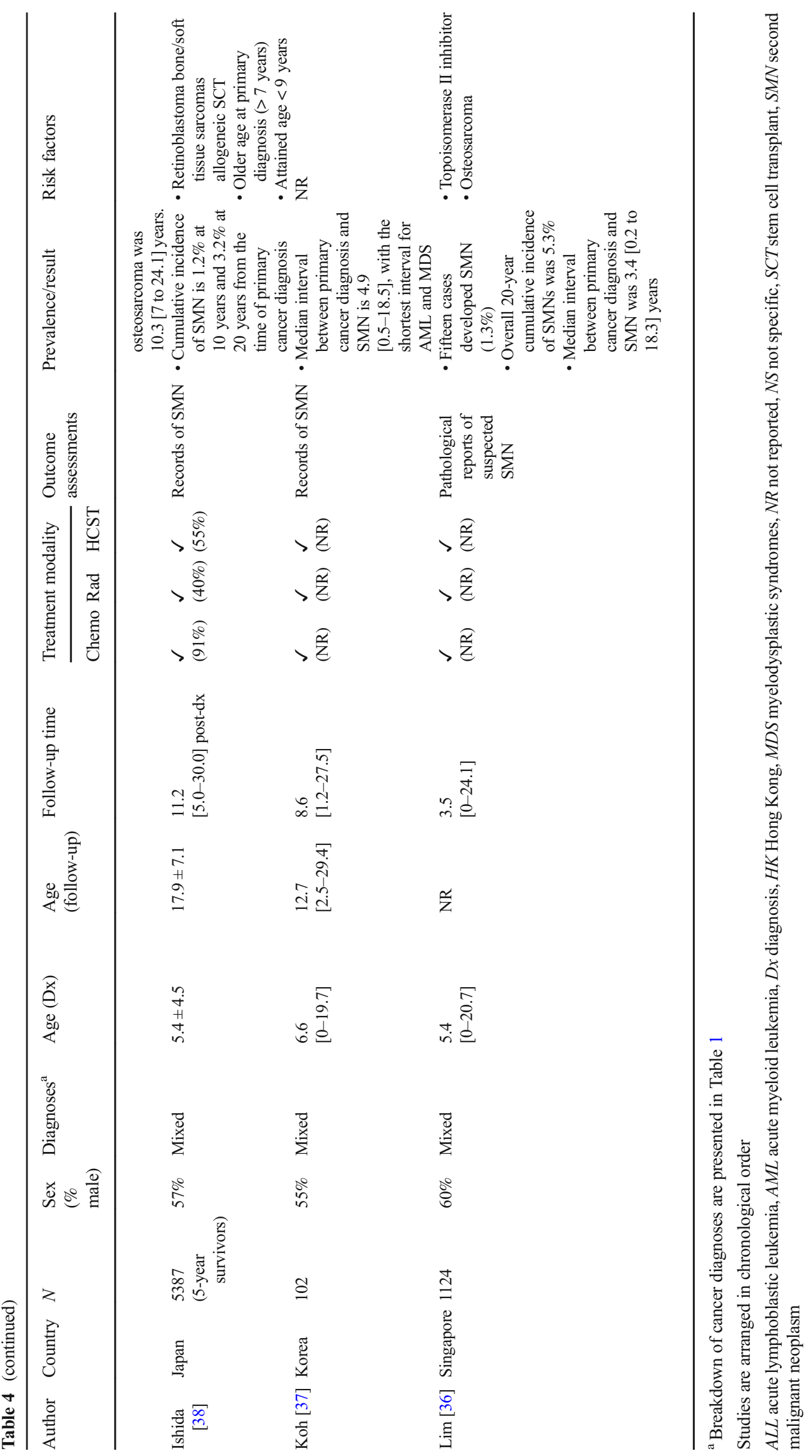


$[113,123,124]$. To address the relevant therapy-related health risks, counseling also promotes culturally relevant protective health behaviors and aims to reduce harmful health behaviors. Such practices are less commonly applied in Asia, and this is complicated by a lack of patient-friendly resources on late effects available in the patients' native languages, especially in developing Asian countries. Personalized care requires providing relevant educational resources that will allow oncology practitioners to address the health effects of treatment. Advances in technology and teleoncology programs may increase both the access to and quality of clinical cancer care through collaborative efforts among medical centers, academic institutions, and NGOs [138]. One viable method involves the use of Internet-based, mobile health, and other innovations to disseminate vital educational information to survivors in developing countries and deprived regions throughout Asia. Given the burden of diseases in these developing countries, the successful application of technology to empower patients during their transition from active treatment to survivorship may potentially have a substantial societal effect.

\section{Limitations}

Although this review has several strengths, such as the inclusion of studies with objective assessments of clinical outcomes and clear definitions of study samples, a few limitations are worth noting. Based on the present studies, it is difficult to estimate the overall prevalence of specific late toxicities in Asia, given the heterogeneity of the study populations and the lack of standardized assessments and definitions of clinical endpoints. Several studies were based on findings from single institutions within a single country. Hence, the treatment characteristics of those study populations may be different from those conducted at other institutions. For example, one included study reported a 5-year cumulative SMN incidence after osteosarcoma of $14.1 \%$ in Singapore, which is considerably higher than the rates reported in other countries, as well as at a medical center in the USA that used a similar treatment protocol for osteosarcoma [36]. However, this single-centered data may be an overestimation of SMN incidence and the authors themselves concluded that this concerning observation warrants further studies of risk factors that might be specific to their population.

As the majority of the included studies are research-driven, there is a potential surveillance bias between the study population and the general childhood cancer population, especially for asymptomatic or subclinical conditions that may be captured by screening and in-depth clinical assessment in a research study, but not in routine clinical setting. It is likely that survivors participating in research studies were more closely assessed over time leading to an overestimation of the prevalence of late effects. Finally, our systematic review found no prevalence data for most Asian countries, including mainland China, Bangladesh and Mongolia, although this may be attributable to the inclusion of only studies and abstracts written in English, as it is methodologically difficult to conduct search and translation processes for literature written in the multiple languages represented in Asia. A formal meta-analysis was also not conducted because the above limitations would severely restrict the ability to draw meaningful conclusions.

\section{Conclusion}

The continued development of national and international collaborative initiatives will yield steady advances in the treatment of childhood cancers throughout Asia over the next few decades. Although additional research regarding childhood cancer treatment in Asia is unquestionably necessary, efforts should also target the emerging population of survivors, particularly in terms of survivorship care. Innovative and collaborative strategies are needed to improve access to quality survivorship care, especially in mainland China and other developing countries in Asia.

Preparation for the impending demands of an emerging population of childhood cancer survivors should begin now. The collective evidence generated by this review, as well as existing literature, may contribute to emerging collaborative research throughout Asia and thus address the needs of survivors on a regional scale. Collaborative initiatives, which will be facilitated by the construction of a regional repository of systematically assessed health outcomes and biospecimens, is expected to facilitate impactful studies of treatment-related, social-environmental and genetic predictors and interventions for late effects in this population.

Funding This study was funded by the Food and Health Bureau Hong Kong under the Health and Medical Research Fund (Grant number 03170047, awarded to Cheung YT).

\section{Compliance with ethical standards}

Conflict of interest Author Long Hin Jonathan Poon declares that he has no conflict of interest.

Author Chun-pong Yu declares that he has no conflict of interest. Author Liwen Peng declares that she has no conflict of interest.

Author Celeste Ewig declares that she has no conflict of interest. Author Hui Zhang declares that he has no conflict of interest.

Author Chi-kong Li declares that he has no conflict of interest.

Author Yin Ting Cheung declares that she has no conflict of interest.

Ethical approval This article does not contain any studies with human participants or animals performed by any of the authors.

This is a systematic review that does not involve any contact with human participants. No informed consent is needed. 
Open Access This article is distributed under the terms of the Creative Commons Attribution 4.0 International License (http:// creativecommons.org/licenses/by/4.0/), which permits unrestricted use, distribution, and reproduction in any medium, provided you give appropriate credit to the original author(s) and the source, provide a link to the Creative Commons license, and indicate if changes were made.

\section{References}

1. Rodriguez-Galindo C, Friedrich P, Alcasabas P, Antillon F, Banavali S, Castillo L, et al. Toward the cure of all children with Cancer through collaborative efforts: pediatric oncology as a global challenge. J Clin Oncol. 2015;33:3065-73.

2. Ferlay J, Soerjomataram I, Dikshit R, Eser S, Mathers C, Rebelo $\mathrm{M}$, et al. Cancer incidence and mortality worldwide: sources, methods and major patterns in GLOBOCAN 2012. Int J Cancer. 2015;136:E386.

3. Nakata K, Ito Y, Magadi W, Bonaventure A, Stiller CA, Katanoda $\mathrm{K}$, et al. Childhood cancer incidence and survival in Japan and England: a population-based study (1993-2010). Cancer Sci. 2018;109:422-34.

4. Park HJ, Moon E, Yoon JY, Oh C, Jung K, Park BK, et al. Incidence and survival of childhood Cancer in Korea. Cancer Res Treat. 2016;48:869-82.

5. Aung L, Khyne T, Yeoh AEJ, Quah TC, Tan AM. A report from the Singapore Childhood Cancer Survivor Study (SG-CCSS): a multi-institutional collaborative study on long-term survivors of childhood cancer, initial analysis reporting for the SG-CCSS. Annals of the Academy of Medicine, Singapore 2009;38:684.

6. Transplant \& Leukemia Survival Rates (1997-2014): Children's Cancer Centre iof the KK Hospital. URL: https://www.kkh.com. sg/about-kkh/corporate-profile/clinical-outcomes/pages/ transplant-leukemia-survival-rates.aspx. Accessed on 1 July 2018.

7. Gupta S, Howard S, Hunger S, Antillon F, Metzger M, et. al."Treating Childhood Cancers in Low- and Middle-Income Countries". In: Disease Control Priorities (third edition): Volume 3, Cancer, edited by H. Gelband, P. Jha, R. Sankaranarayanan, S. Horton. Washington, DC: World Bank.\&nbsp; .

8. Howard SC, Zaidi A, Cao X, Weil O, Bey P, Patte C, et al. The my child matters programme: effect of public-private partnerships on paediatric cancer care in low-income and middle-income countries. Lancet Oncol. 2018;19:e266.

9. Lu, L., Huang, C., Huang,H. Childhood cancer: an emerging public health issue in China. Annf Transl Med 2015;3.

10. Zheng, Rongshou|Peng, Xiaoxia|Zeng, Hongmei|Zhang, Siwei|Chen, Tianhui|Wang, Huanmin|Chen, Wanqing. Incidence, mortality and survival of childhood cancer in China during 2000-2010 period: a population-based study. Cancer Lett 2015;363:176-180.

11. Bidwell SS, Peterson CC, Demanelis K, Zarins KR, Meza R, Sriplung $\mathrm{H}$ et al. Childhood cancer incidence and survival in Thailand: A comprehensive population-based registry analysis, 1990-2011. Pediatric Blood \& Cancer 2019;66:n/a.

12. Hudson MM, Ness KK, Gurney JG, Mulrooney DA, Chemaitilly W, Krull KR, et al. Clinical ascertainment of health outcomes among adults treated for childhood cancer. JAMA. 2013;309: 2371-81.

13. Fidler MM, Reulen RC, Winter DL, Kelly J, Jenkinson HC, Skinner R, et al. Long term cause specific mortality among 34 489 five year survivors of childhood cancer in Great Britain: population based cohort study. The BMJ. 2016;354:i4351.

14. Henderson TO, Ness KK, Cohen HJ. Accelerated aging among cancer survivors: from pediatrics to geriatrics. Am Soc Clin Oncol Educ Book. 2014;423.
15. Oeffinger KC, Mertens AC, Sklar CA, Kawashima T, Hudson MM, Meadows AT, et al. Chronic health conditions in adult survivors of childhood Cancer. N Engl J Med. 2006;355:1572-82.

16. Hewitt M, Greenfield S, Stovall E. From Cancer patient to Cancer survivor: lost in transition. 2006.

17. SIOP Asia (International Society of Paediatric Oncology). URL: https://siop-online.org/sp_cb/asia/. Accessed on 1 July 2018.

18. Childhood Cancer International. URL: https://www. childhoodcancerinternational.org/cci-global-network/asia/. Accessed on 1 July 2018.

19. Working Together to Address Complex health Challenges: Association of Southeast Asian Nations. URL: https://asean.org/ asean-socio-cultural/asean-health-ministers-meeting-ahmm/ overview-2/. Accessed on 1 July 2018.

20. United Nations Regional Groups of Member States: the United Nations. URL: http://www.un.org/depts/DGACM/ RegionalGroups.shtml. Accessed on 1 July 2018.

21. Taylor N, Absolom K, Michel G, Urquhart T, Gerrard M, Jenkins A, et al. Comparison of self-reported late effects with medical records among survivors of childhood cancer. Eur J Cancer. 2010;46:1069-78.

22. National Heart, Lung and Blood Institute. Quality Assessment Tool for Observational Cohort and Cross-Sectional Studies. URL: https://www.nhlbi.nih.gov/health-topics/study-qualityassessment-tools. Accessed on 1 July 2018.

23. Viera AJ, Garrett JM. Understanding interobserver agreement: the kappa statistic. Fam Med. 2005;37:360.

24. Han JW, Kim HS, Kim BS, Kwon SY, Shin YJ, Kim SH, et al. Increasing and worsening late effects in childhood Cancer survivors during follow-up. J Korean Med Sci. 2013;28:755-62.

25. Pakakasama S, Veerakul G, Sosothikul D, Chainansamit S-o, Laosombat V, Thanarattanakorn P, et al. Late effects in survivors of childhood acute lymphoblastic leukemia: a study from Thai pediatric oncology group. Int J Hematol. 2010;91:850-4.

26. Han JW, Kwon SY, Won SC, Shin YJ, Ko JH, Lyu CJ. Comprehensive clinical follow-up of late effects in childhood cancer survivors shows the need for early and well-timed intervention. Ann Oncol. 2009;20:1170-7.

27. Matsuzaki A, Ishii E, Nagatoshi Y, Eguchi H, Koga H, Yanai F, et al. Long-term outcome of treatment with protocols AL841, AL851, and ALHR88 in children with acute lymphoblastic leukemia: results obtained by the Kyushu-Yamaguchi Children's Cancer study group. Int J Hematol. 2001;73:369-77.

28. Azanan MS, Abdullah NK, Chua LL, Lum SH, Abdul Ghafar SS, Kamarulzaman A, et al. Immunity in young adult survivors of childhood leukemia is similar to the elderly rather than agematched controls: role of cytomegalovirus. Eur J Immunol. 2016;46:1715-26.

29. Mahmoud SSA, Fukushima T, Shimizu T, Nakao T, Yamaguchi R, Fukushima H, et al. Persistent low level Epstein-Barr virus DNAemia in childhood Cancer survivors. Pediatr Hematol Oncol. 2013;30:216-25.

30. Watanabe Nemoto M, Isobe K, Togasaki G, Kanazawa A, Kurokawa M, Saito M, et al. Delayed renal dysfunction after total body irradiation in pediatric malignancies. J Radiat Res. 2014;55: 996-1001.

31. Yoo S, Kim JH, Eo H, Jeon TY, Sung KW, Kim HS. Dynamic MRI findings and clinical features of benign hypervascular hepatic nodules in childhood-cancer survivors. AJR Am J Roentgenol. 2013;201:178-84.

32. Lee MH, Yoo S, Kim JH, Eo H, Jeon TY, Kim SH, et al. Hypervascular hepatic nodules in childhood cancer survivors: clinical and imaging features. Clin Imaging. 2012;36:301-7.

33. Tanaka M, Kamata T, Yanagisawa R, Morita D, Saito S, Sakashita $\mathrm{K}$, et al. Increasing risk of disturbed root development in 
permanent teeth in childhood Cancer survivors undergoing Cancer treatment at older age. J Pediatr Hematol Oncol. 2017;1.

34. Kang C, Hahn SM, Kim HS, Lyu CJ, Lee J, Lee J et al. Clinical risk factors influencing dental developmental disturbances in childhood Cancer survivors. J Korean Cancer Assoc 2017;0:0.

35. Nishimura $\mathrm{S}$, Inada $\mathrm{H}$, Sawa $\mathrm{Y}$, Ishikawa $\mathrm{H}$. Risk factors to cause tooth formation anomalies in chemotherapy of paediatric cancers. Eur J Cancer Care. 2013;22:353-60.

36. Lim JW, Yeap FS, Chan YH, Yeoh AE, Quah TC, Tan PL. Second malignant neoplasms in childhood Cancer survivors treated in a tertiary Paediatric oncology Centre. Ann Acad Med Singap. 2017;46:11-9.

37. Koh K, Yoo KH, Im HJ, Sung KW, Koo HH, Kim HS, et al. Characteristics and outcomes of second malignant neoplasms after childhood Cancer treatment: multi-center retrospective survey. J Korean Med Sci. 2016;31:1254-61.

38. Ishida Y, Qiu D, Maeda M, Fujimoto J, Kigasawa H, Kobayashi $\mathrm{R}$, et al. Secondary cancers after a childhood cancer diagnosis: a nationwide hospital-based retrospective cohort study in Japan. Int J Clin Oncol. 2016;21:506-16.

39. Fujiwara T, Fujiwara M, Numoto K, Ogura K, Yoshida A, Yonemoto $\mathrm{T}$, et al. Second primary osteosarcomas in patients with retinoblastoma. Jpn J Clin Oncol. 2015;45:1139.

40. Ishida Y, Maeda M, Urayama KY, Kiyotani C, Aoki Y, Kato Y, et al. Secondary cancers among children with acute lymphoblastic leukaemia treated by the Tokyo Children's Cancer study group protocols: a retrospective cohort study. Br J Haematol. 2014;164:101-12.

41. Sun WF, Cheng FW, Lee V, Leung WK, Shing MK, Yuen PM, et al. Second malignant neoplasms in childhood cancer survivors in a tertiary paediatric oncology Centre in Hong Kong, China. Chin Med J. 2011;124:3686-92.

42. Araki Y, Matsuyama Y, Kobayashi Y, Toyokawa S, Inoue K, Suzuki S, et al. Secondary neoplasms after retinoblastoma treatment: retrospective cohort study of 754 patients in Japan. Jpn J Clin Oncol. 2011;41:373-9.

43. Yamasaki F, Takayasu T, Nosaka R, Nishibuchi I, Kawaguchi H, Kolakshyapati M, et al. Development of cystic malacia after highdose cranial irradiation of pediatric CNS tumors in long-term follow-up. Childs Nerv Syst. 2017;33:957-64.

44. Tay CG, Lee VWM, Ong LC, Goh KJ, Ariffin H, Fong CY. Vincristine-induced peripheral neuropathy in survivors of childhood acute lymphoblastic leukaemia. Pediatr Blood Cancer 2017: e26471, 64.

45. Yamasaki F, Takayasu T, Nosaka R, Kenjo M, Akiyama Y, Tominaga A, et al. The postirradiation incidence of cavernous angioma is higher in patients with childhood pineoblastoma or primitive neuroectodermal tumors than medulloblastoma. Childs Nerv Syst. 2015;31:901-7.

46. Kim SJ, Park MH, Lee JW, Chung NG, Cho B, Lee IG, et al. Neurocognitive outcome in survivors of childhood acute lymphoblastic leukemia: experience at a tertiary Care Hospital in Korea. J Korean Med Sci. 2014;30:463-9.

47. Liang S, Yang T, Chen Y, Liang M, Chen H, Chang K, et al. Neuropsychological functions and quality of life in survived patients with intracranial germ cell tumors after treatment. Neurooncology. 2013;15:1543-51

48. Chiou S, Jang R, Liao Y, Yang P. Health-related quality of life and cognitive outcomes among child and adolescent survivors of leukemia. Support Care Cancer. 2010;18:1581-7.

49. Kikuchi A, Maeda M, Hanada R, Okimoto Y, Ishimoto K, Kaneko $\mathrm{T}$, et al. Moyamoya syndrome following childhood acute lymphoblastic leukemia. Pediatr Blood Cancer. 2007;48:268-72.

50. Khong PL, Leung LH, Fung AS, Fong DY, Qiu D, Kwong DL et al. White Matter Anisotropy in Post-Treatment Childhood
Cancer Survivors: Preliminary Evidence of Association With Neurocognitive Function. J Clin Oncol 2006;24:884-890.

51. Khong PL, Leung LH, Chan GC, Kwong DL, Wong WH, Cao G et al. White matter anisotropy in childhood medulloblastoma survivors: association with neurotoxicity risk factors. Radiology 2005;236:647-652.

52. Khong PL, Kwong DL, Chan GC, Sham JS, Chan FL, Ooi G. Diffusion-tensor imaging for the detection and quantification of treatment-induced white matter injury in children with Medulloblastoma: a pilot study. Am J Neuroradiol. 2003;24:734.

53. Yoon JY, Park HJ, Ju HY, Yoon JH, Chung JS, Hwang SH, et al. Gonadal and sexual dysfunction in childhood Cancer survivors. Cancer Res Treat. 2017;49:1057-64.

54. Adachi M, Oto Y, Muroya K, Hanakawa J, Asakura Y, Goto H. Partial lipodystrophy in patients who have undergone hematopoietic stem cell transplantation during childhood: an institutional cross-sectional survey. Clin Pediatr Endocrinol. 2017;26:99-108.

55. Lee HJ, Hahn SM, Jin SL, Shin YJ, Kim SH, Lee YS, et al. Subclinical hypothyroidism in childhood Cancer survivors. Yonsei Med J. 2016;57:915-22.

56. Han JW, Kim HS, Hahn SM, Jin SL, Shin YJ, Kim SH, et al. Poor bone health at the end of puberty in childhood cancer survivors. Pediatr Blood Cancer. 2015;62:1838-43.

57. Kojima C, Kubota M, Nagai A, Adachi S, Watanabe K, Nakahata T. Adipocytokines in childhood cancer survivors and correlation with metabolic syndrome components. Pediatr Int. 2013;55:438-42.

58. Choi YJ, Park SY, Cho WK, Lee JW, Cho KS, Park SH, et al. Factors related to decreased bone mineral density in childhood Cancer survivors. J Korean Med Sci. 2013;28:1632-8.

59. Miyoshi Y, Ohta H, Namba N, Tachibana M, Miyamura T, Miyashita E, et al. Low serum concentrations of anti-Müllerian hormone are common in 53 female childhood Cancer survivors. Horm Res Paediat. 2013;79:17-21.

60. Kang M, Kim S, Lee Y, Shin C, Yang S, Lim J. Risk factors for osteoporosis in long-term survivors of intracranial germ cell tumors. Osteoporos Int. 2012;23:1921-9.

61. Hyodo H, Ishiguro H, Tomita Y, Takakura H, Koike T, Shimizu T, et al. Decreased serum testosterone levels in long-term adult survivors with fatty liver after childhood stem cell transplantation. Biol Blood Marrow Transplant. 2012;18:1119-27.

62. Sohn YB, Kim SJ, Park SW, Kim S, Cho S, Lee SH, et al. The metabolic syndrome and body composition in childhood cancer survivors. Korean J Pediatr. 2011;54:253-9.

63. Nishi Y, Hamamoto K, Fujita N, Okada S. Empty Sella/pituitary atrophy and endocrine impairments as a consequence of radiation and chemotherapy in long-term survivors of childhood leukemia. Int J Hematol. 2011;94:399-402.

64. Tomita Y, Ishiguro H, Yasuda Y, Hyodo H, Koike T, Shimizu T, et al. High incidence of fatty liver and insulin resistance in longterm adult survivors of childhood SCT. Bone Marrow Transplant. 2011;46:416-25.

65. Lusawat A, Dhiravibulya K, Thammongkol S, Vanavanan S, Mahachoklertwattana P. Hypothalamic-pituitary dysfunction in survivors of childhood brain tumors in Prasat neurological institute. J Med Assoc Thai. 2010;93:38-47.

66. Surapolchai P, Hongeng S, Mahachoklertwattana P, Pakakasama $\mathrm{S}$, Winaichatsak A, Wisanuyothin N, et al. Impaired glucose tolerance and insulin resistance in survivors of childhood acute lymphoblastic leukemia: prevalence and risk factors. J Pediatr Hematol Oncol. 2010;32:383-9.

67. Adachi M, Muroya K, Asakura Y. Unfavorable lipoprotein profile in childhood cancer survivors with suprasellar brain tumors - a high Apo B level and increased small dense LDL-cholesterol. Childs Nerv Syst. 2009;25:669-75. 
68. Miyoshi Y, Ohta H, Hashii Y, Tokimasa S, Namba N, Mushiake S, et al. Endocrinological analysis of 122 Japanese childhood Cancer survivors in a single hospital. Endocr J. 2008;55:1055-63.

69. Ishiguro H, Yasuda Y, Tomita Y, Shinagawa T, Shimizu T, Morimoto T, et al. Gonadal shielding to irradiation is effective in protecting testicular growth and function in long-term survivors of bone marrow transplantation during childhood or adolescence. Bone Marrow Transplant. 2007;39:483-90.

70. Jaruratanasirikul S, Owasith K, Wongchanchailert M, Laosombat V, Sriplung H. Growth patterns and final height of survivors of childhood leukemia. J Pediatr Endocrinol Metab. 2004;17:719.

71. Yamashita N, Tanaka H, Moriwake T, Nishiuchi R, Oda M, Seino Y. Analysis of linear growth in survivors of childhood acute lymphoblastic leukemia. J Bone Miner Metab. 2003;21:172-8.

72. Li VW, Lai CT, Liu AP, Cheuk DK, Cheung Y. Left Atrial Mechanics and Integrated Calibrated Backscatter in Anthracycline-Treated Long-Term Survivors of Childhood Cancers. Ultrasound Med Biol 2017.

73. Li VW, Cheuk DK, Cheng FW, Yang JY, Yau JP, Ho KK, et al. Myocardial stiffness as assessed by diastolic wall strain in adult survivors of childhood leukaemias with preserved left ventricular ejection fraction. Eur Heart J Cardiovasc Imaging. 2017;18:451-8.

74. Cheung Y, Lam WW, Ip JJ, Cheuk DK, Cheng FW, Yang JY, et al. Myocardial iron load and fibrosis in long term survivors of childhood leukemia. Pediatr Blood Cancer. 2015;62:698-703.

75. Yu H, Yu W, Cheuk DKL, Wong SJ, Chan GCF, Cheung Y. New three-dimensional speckle-tracking echocardiography identifies global impairment of left ventricular mechanics with a high sensitivity in childhood Cancer survivors. J Am Soc Echocardiogr. 2013;26:846-52.

76. Yu W, Li S, Chan GCF, Ha S, Wong SJ, Cheung Y. Transmural strain and rotation gradient in survivors of childhood cancers. Eur Heart J Cardiovasc Imaging. 2013;14:175-82.

77. Cheung YF, Yu W, Cheuk DKL, Cheng WF, Yang YJ, Yau PJ, et al. Plasma high sensitivity troponin T levels in adult survivors of childhood Leukaemias: determinants and associations with cardiac function. PLoS One. 2013;8:e77063.

78. Shimomura Y, Baba R, Watanabe A, Horikoshi Y, Asami K, Hyakuna N, et al. Assessment of late cardiotoxicity of pirarubicin (THP) in children with acute lymphoblastic leukemia. Pediatr Blood Cancer. 2011;57:461-6.

79. Cheung Y, Li S, Chan GCF, Wong SJ, Ha S. Left ventricular twisting and untwisting motion in childhood Cancer survivors. Echocardiography. 2011;28:738-45.

80. Hamada H, Ohkubo T, Maeda M, Ogawa S. Evaluation of cardiac reserved function by high-dose dobutamine-stress echocardiography in asymptomatic anthracycline-treated survivors of childhood cancer. Pediatr Int. 2006;48:313-20.

81. Chan M, Roebuck DJ, Yuen M, Li C, Chan Y. MR imaging of the brain in patients cured of acute lymphoblastic leukemia-the value of gradient Echo imaging. AJNR Am J Neuroradiol. 2006;27:548-52.

82. Chan Y, Roebuck DJ, Yuen M, Yeung K, Lau K, Li C, et al. Longterm cerebral metabolite changes on proton magnetic resonance spectroscopy in patients cured of acute lymphoblastic leukemia with previous intrathecal methotrexate and cranial irradiation prophylaxis. Int J Radiat Oncol Biol Phys. 2001;50:759-63.

83. Yu AF, Raikhelkar J, Zabor EC, Tonorezos ES, Moskowitz CS, Adsuar R, et al. Two-dimensional speckle tracking echocardiography detects subclinical left ventricular systolic dysfunction among adult survivors of childhood, adolescent, and young adult Cancer. Biomed Res Int. 2016;2016:9363951.

84. Thavendiranathan P, Poulin F, Lim K, Plana JC, Woo A, Marwick TH. Use of myocardial strain imaging by echocardiography for the early detection of cardiotoxicity in patients during and after Cancer chemotherapy: a systematic review. J Am Coll Cardiol. 2014;63:2751-68.
85. Mor-Avi V, Lang RM, Badano LP, Belohlavek M, Cardim NM, Derumeaux G, et al. Current and evolving echocardiographic techniques for the quantitative evaluation of cardiac mechanics: ASE/ EAE consensus statement on methodology and indications endorsed by the Japanese Society of Echocardiography. Eur J Echocardiogr. 2011;12:167-205.

86. Wojnowski L, Kulle B, Schirmer M, Schluter G, Schmidt A, Rosenberger A, et al. NAD(P)H oxidase and multidrug resistance protein genetic polymorphisms are associated with doxorubicininduced cardiotoxicity. Circulation. 2005;112:3754-62.

87. Mostoufi-Moab S, Seidel K, Leisenring WM, Armstrong GT, Oeffinger KC, Stovall M, et al. Endocrine abnormalities in aging survivors of childhood Cancer: a report from the childhood Cancer survivor study. J Clin Oncol. 2016;34:3240-7.

88. Ramachandran A, Snehalatha C. Rising burden of obesity in Asia. J Obes. 2010;2010:1-8.

89. Cheung Y. Cancer Pharmacoethnicity: ascertaining chronic health outcomes in survivors of childhood Cancer in Asia. Glob J Pharmaceu Sci. 2017;4:555634.

90. Syn NL, Yong WP, Lee SC, Goh BC. Genetic factors affecting drug disposition in Asian cancer patients. Expert Opin Drug Metab Toxicol. 2015;11:1879-92.

91. Peter H. O'Donnell, M. Eileen Dolan. Cancer Pharmacoethnicity: ethnic differences in susceptibility to the effects of chemotherapy. Clin Cancer Res. 2009;15:4806-14.

92. Phan VH, Moore MM, McLachlan AJ, Piquette-Miller M, Xu H, Clarke SJ. Ethnic differences in drug metabolism and toxicity from chemotherapy. Expert Opin Drug Metab Toxicol. 2009;5:243-57.

93. Brooke R, Im C, L Wilson C, J Krasin M, Liu Q, Li Z et al. A High-risk Haplotype for Premature Menopause in Childhood Cancer Survivors Exposed to Gonadotoxic Therapy, J Natl Cancer Inst 2018;110(8):895-904.

94. Nishimura A, Nonomura H, Tanaka S, Yoshida M, Maruyama Y, Aritomi Y, et al. Characterization of APOE and TOMM40 allele frequencies in the Japanese population. Alzheimers Dement. 2017;3:524-30.

95. Farrer LA, Cupples LA, Haines JL, Hyman B, Kukull WA, Mayeux R, et al. Effects of age, sex, and ethnicity on the association between apolipoprotein E genotype and Alzheimer disease. A meta-analysis. APOE and Alzheimer disease Meta analysis consortium. JAMA. 1997;278:1349-56.

96. Belle FN, Weiss A, Schindler M, Goutaki M, Bochud M, Zimmermann K, et al. Overweight in childhood cancer survivors: the Swiss Childhood Cancer Survivor Study. Am J Clin Nutr. 2018;107(1):3-11.

97. Mulrooney DA, Ness KK, Neglia JP, Whitton JA, Green DM, Zeltzer LK, et al. Fatigue and sleep disturbance in adult survivors of childhood Cancer: a report from the childhood Cancer survivor study (CCSS). Sleep. 2007;31:271-81.

98. Cheung YT, Brinkman TM, Mulrooney DA, Mzayek Y, Liu W, Banerjee $\mathrm{P}$, et al. Impact of sleep, fatigue, and systemic inflammation on neurocognitive and behavioral outcomes in long-term survivors of childhood acute lymphoblastic leukemia. Cancer. 2017;123:3410-9.

99. Li HC, Lopez V, Joyce Chung OK, Ho KY, Chiu SY. The impact of cancer on the physical, psychological and social well-being of childhood cancer survivors. Eur J Oncol Nurs. 2013;17:214-9.

100. Bode AM, Wang ZDH. Cancer prevention and control: alarming challenges in China. Natl Sci Rev. 2016;3:117-27.

101. Leisenring WM, Mertens AC, Armstrong GT, Stovall MA, Neglia JP, Lanctot JQ, et al. Pediatric cancer survivorship research: experience of the childhood Cancer survivor study. J Clin Oncol. 2009;27:2319-27.

102. Grbner SN, Worst BC, Weischenfeldt J, Buchhalter I, Kleinheinz $\mathrm{K}$, Rudneva VA, et al. The landscape of genomic alterations across childhood cancers. Nature. 2018;555:321-7. 
103. deAndrés-Galiana EJ, Fernández-Martínez JL, Luaces O, del Coz JJ, Huergo-Zapico L, Acebes-Huerta A, et al. Analysis of clinical prognostic variables for chronic lymphocytic leukemia decisionmaking problems. J Biomed Inform. 2016;60:342-51.

104. Yang J, Landier W, Yang W, Liu C, Hageman L, Cheng C, et al. Inherited NUDT15 variant is a genetic determinant of mercaptopurine intolerance in children with acute lymphoblastic leukemia. J Clin Oncol. 2015;33:1235-42.

105. Moriyama T, Nishii R, Perez-Andreu V, Yang W, Klussmann FA, Zhao X, et al. NUDT15 polymorphisms alter thiopurine metabolism and hematopoietic toxicity. Nat Genet. 2016;48:367-73.

106. Qian M, Cao X, Devidas M, Yang W, Cheng C, Dai Y, et al. TP53 germline variations influence the predisposition and prognosis of B-cell acute lymphoblastic leukemia in children. J Clin Oncol. 2018;36:591-9.

107. Roberts KG, Li Y, Payne-Turner D, Harvey RC, Yang Y, Pei D, et al. Targetable kinase-activating lesions in Ph-like acute lymphoblastic leukemia. N Engl J Med. 2014;371:1005-15.

108. Watt L, Gulati S, Shaw N, Sung L, Dix D, Poureslami I, et al. Perceptions about complementary and alternative medicine use among Chinese immigrant parents of children with cancer. Support Care Cancer. 2012;20:253-60.

109. Hamidah A, Rustam ZA, Tamil AM, Zarina LA, Zulkifli ZS, Jamal R. Prevalence and parental perceptions of complementary and alternative medicine use by children with cancer in a multiethnic southeast Asian population. Pediatr Blood Cancer. 2009;52:70-4.

110. Chung VCH, Ma PHX, Lau CH, Wong SYS, Yeoh EK, Griffiths SM. Views on traditional Chinese medicine amongst Chinese population: a systematic review of qualitative and quantitative studies. Health Expect. 2014;17:622-36.

111. Radossi A, Taromina K, Marjerrison S, Diorio C, Similio R, Njuguna F, et al. A systematic review of integrative clinical trials for supportive care in pediatric oncology: a report from the International Society of Pediatric Oncology, T\&CM collaborative. Support Care Cancer. 2018;26:375-91.

112. McClafferty H, Vohra S, Bailey M, Brown M, Esparham A, Gerstbacher D, et al. Pediatric Integrative Medicine. Pediatrics. 2017;140:e20171961.

113. McCabe MS, Partridge A, Grunfeld E, Hudson MM. Risk-based health care, the Cancer survivor, the oncologist and the primary care physician. Semin Oncol. 2013;40:804-12.

114. Landier W, Bhatia S, Eshelman DA, Forte KJ, Sweeney T, Hester $\mathrm{AL}$, et al. Development of risk-based guidelines for pediatric cancer survivors: the Children's oncology group long-term follow-up guidelines from the Children's oncology group late effects committee and nursing discipline. J Clin Oncol. 2004;22:4979-90.

115. The Children's Oncology Group Long-Term Follow-Up Guidelines for Survivors of Childhood, Adolescent, and Young Adult Cancers. Version.4. 2013.

116. Ponsford J. International growth of neuropsychology. Neuropsychology. 2017;31:921-33.

117. Sakamoto M. Neuropsychology in Japan: history, current challenges, and future prospects. Clin Neuropsychol. 2016;30:1278-95.

118. Chan AS, Sze SL, Cheung M, Han YMY. Development and application of neuropsychology in Hong Kong: implications of its value and future advancement. Clin Neuropsychol. 2016;30:1236-51.

119. Hewitt M. Childhood Cancer survivorship : improving care and quality of life. Washington: National Academies Press; 2003.

120. Oeffinger $\mathrm{KC}, \mathrm{McC}$ abe MS. Models for delivering survivorship care. JCO. 2006;24:5117-24.

121. Chan A, Lum ZK, Ng T, Eyob T, Wang XJ, Chae J, et al. Perceptions and barriers of survivorship Care in Asia: perceptions from Asian breast Cancer survivors. J Global Oncol. 2016;3:104.
122. Yasushi I, Mari T, Mitsue H, Fumiko I. Japanese childhood cancer survivors' readiness for care as adults: a cross-sectional survey using the transition scales. Psycho-Oncology. 2017;26:1019-26.

123. Poplack DG, Fordis M, Landier W, Bhatia S, Hudson MM, Horowitz ME. Childhood cancer survivor care: development of the passport for care. Nat Rev Clin Oncol. 2014;11:740-50.

124. Landier W, Chen Y, Namdar G, Francisco L, Wilson K, Herrera C, et al. Impact of tailored education on awareness of personal risk for therapy-related complications among childhood Cancer survivors. J Clin Oncol. 2015;33:3887-93.

125. Li WHC, Ho KY, Ho LLK, Lam HS, Lam KKW, Chui SY, et al. Adventure-based training to promote physical activity and reduce fatigue among childhood cancer survivors: a randomized controlled trial. Int J Nurs Stud. 2018;83:65-74.

126. Cheung AT, Li WHC, Ho KY, Lam KKW, Ho LLK, Chiu SY, et al. Efficacy of musical training on psychological outcomes and quality of life in Chinese pediatric brain tumor survivors. PsychoOncology. 2019;28:174-80.

127. Ishida Y, Takahashi M, Maru M, Mori M, Henderson TO, Daugherty CK, et al. Physician preferences and knowledge regarding the Care of Childhood Cancer Survivors in Japan: a mailed survey of the Japanese Society of Pediatric Oncology. Jpn J Clin Oncol. 2012;42:513-21.

128. Miura A, Matsuda Y, Ogawa I, Takagai J, Hirai K, Hosoda Y, et al. Oncology nurses' recognition of long-term cancer survivorship care in Japan. Asia Pac J Oncol Nurs. 2015;2:136.

129. Ang SH, Koh SSL, Lee, Xiu Hua @ Hideka Tamamura, Shorey S. Experiences of adolescents living with cancer: a descriptive qualitative study. J Child Health Care 2018;22:532-544.

130. Park HJ. Long-term follow-up study and long-term care of childhood cancer survivors. Korean J Pediatr. 2010;53:465-70.

131. Ghim TT. Time to establish multidisciplinary childhood cancer survivorship programs in Korea. Korean J Hematol. 2010;45: 84-7.

132. Yuza Y. Long-term follow-up program and transition medicine for childhood cancer survivors. Gan to kagaku ryoho Cancer \& chemotherapy. 2014;41:15.

133. Ahn E, Park HJ, Baek HJ, Hwang PH, Lee YH, Park B, et al. Awareness about past diagnosis and treatment history: nationwide survey of childhood cancer survivors and their parents. Jpn J Clin Oncol. 2017;47:962-8.

134. Hopkins J, Burns E, Eden T. International twinning partnerships: An effective method of improving diagnosis, treatment and care for children with cancer in low-middle income countries. J Cancer Policy 2013;1:e19.

135. Chung OK, Li HC, Chiu SY, Ho KY, Lopez V. The impact of cancer and its treatment on physical activity levels and behavior in Hong Kong Chinese childhood cancer survivors. Cancer Nurs. 2014;37:43.

136. Kim K, Kim J. Factors influencing health-related quality of life among Korean cancer survivors. Psycho-Oncology. 2017;26:81-7.

137. Park JJ, Park HA. Prevalence of cigarette smoking among adult Cancer survivors in Korea. Yonsei Med J. 2015;56:556-62.

138. Hazin R. MD, Qaddoumi I, Dr. Teleoncology: current and future applications for improving cancer care globally. Lancet Oncol. 2010;11:204-10

Publisher's note Springer Nature remains neutral with regard to jurisdictional claims in published maps and institutional affiliations. 